\title{
Numerical Solution of Mean-Square Approximation Problem of Real Nonnegative Function by the Modulus of Double Fourier Integral
}

\author{
Petro Savenko, Myroslava Tkach \\ Pidstryhach Institute for Applied Problems of Mechanics and Mathematics, Lviv, Ukraine \\ E-mail:spo@iapmm.lviv.ua,tmd@iapmm.lviv.ua \\ Received May 27, 2011; revised June 21, 2011; accepted June 29, 2011
}

\begin{abstract}
A nonlinear problem of mean-square approximation of a real nonnegative continuous function with respect to two variables by the modulus of double Fourier integral dependent on two real parameters with use of the smoothing functional is studied. Finding the optimal solutions of this problem is reduced to solution of the Hammerstein type two-dimensional nonlinear integral equation. The numerical algorithms to find the branching lines and branching-off solutions of this equation are constructed and justified. Numerical examples are presented.
\end{abstract}

Keywords: Mean-Square Approximation, Discrete Fourier Transform, Two-Dimensional Nonlinear Integral Equation, Nonuniqueness and Branching of Solutions, Two-Dimensional Nonlinear Spectral Problem

\section{Introduction}

A variational problem about mean-square approximation of a real finite function by the modulus of double Fourier integral with use of smoothing functional [1] is studied. The nonuniqueness and branching of solutions is an essential feature of nonlinear approximation problem. The problem of finding a set of branching points is insufficiently investigated nonlinear two-parameter spectral problem. The existence of connected components of the spectrum, which in the case of real parameters, similarly as in [2], are spectral lines, is essential difference of two-dimensional spectral problems compared with onedimensional ones.

The algorithms for finding the lines of possible branching of solutions of the Hammerstein type nonlinear equation, which are based on implicit functions methods, are proposed and justified. The algorithms for numerical finding the optimal solutions of the approximation problem are constructed and justified also. Numerical examples are presented.

Note that this class of problems are widely used at solving the inverse problems of radio physics, acoustics and so on $[3,4]$.

\section{Problem Formulation, Basic Equations and Relations}

Consider the linear integral operator

$$
\begin{aligned}
f\left(s_{1}, s_{2}\right) & =A U \\
& \equiv \iint_{G} U(x, y) \exp \left(-i\left(c_{1} x s_{1}+c_{2} y s_{2}\right)\right) \mathrm{d} x \mathrm{~d} y,
\end{aligned}
$$

which is the double Fourier transform of function $U(x, y) \in L_{2}(G)$, dependent on the real two-dimensional parameter $\mathbf{c}=\left(c_{1}, c_{2}\right), \quad 0<c_{i}<\infty \quad(i=1,2)^{1}$. Operator $U$ acts from space $L_{2}(G)$ into the solid angle $L_{2}(\Omega)$, where $\Omega \subset \mathbb{R}^{2}$ is some limited domain in which a real continuous nonnegative and nonzero function $F\left(s_{1}, s_{2}\right)$ is given. In the spaces $L_{2}(G)$ and $L_{2}(\Omega)$ we introduce scalar products and generable by them norms

$$
\begin{aligned}
& \left(U_{1}, U_{2}\right)_{L_{2}(G)}=\frac{4 \pi^{2}}{c_{1} c_{2}} \iint_{G} U_{1}(x, y) \overline{U_{2}(x, y)} \mathrm{d} x \mathrm{~d} y \\
& \|U\|_{L_{2}(G)}=(U, U)_{L_{2}(G)}^{1 / 2},
\end{aligned}
$$

\footnotetext{
${ }^{1}$ Parameters $c_{1}, c_{2}$ are physical parameters of the object being investigated. In particular, in the antennas synthesis problems these parameters characterize the electrical sizes of aperture of radiating system and a solid angle in which the necessary energetic characteristic of radiation is given [4].
} 


$$
\begin{gathered}
\left(f_{1}, f_{2}\right)_{L_{2}(\Omega)}=\iint_{\Omega} f_{1}\left(s_{1}, s_{2}\right) \overline{f_{2}\left(s_{1}, s_{2}\right)} \mathrm{d} s_{1} \mathrm{~d} s_{2}, \\
\|f\|=(f, f)_{L_{2}(\Omega)}^{1 / 2} .
\end{gathered}
$$

Since the domain $G \subset \mathbb{R}^{2}$ is limited, the integral in (1) exists in the usual sense [5] for an arbitrary function $U \in L_{2}(G)$. Here the function $f\left(s_{1}, s_{2}\right)$ is continuous and quadratically integrable.

Consider the problem about approximation of a real continuous and nonnegative function $F\left(s_{1}, s_{2}\right)$ in the domain $\bar{\Omega}$ by the modulus of the Fourier integral (1). We shall formulate it as a minimization problem of the smoothing functional

$$
\begin{aligned}
& \min _{U \in L_{2}(G)} \sigma_{\alpha}(U)=\|F-\mid A U\|_{L_{2}(\Omega)}^{2}+\alpha\|U\|_{L_{2}(G)}^{2} \\
& \equiv\|F-|f|\|_{L_{2}(\Omega)}^{2}+\alpha\|U\|_{L_{2}(G)}^{2} .
\end{aligned}
$$

Here the first summand describes the mean-square deviation of the modulus of the Fourier integral from the given function $F\left(s_{1}, s_{2}\right)$ in the domain $\bar{\Omega}$. The second summand imposes constraints on the norm of the Fourier integral prototype, $\alpha$ is a weight (regularizing) parameter.

Equating the Hato differential of the functional (4) to zero and taking into account (1), we write the equation concerning the function $U$ that describes the fixed points $\sigma_{\alpha}(U)$ in the space $L_{2}(G)$ :

$$
\alpha U=-A^{*} A U+A^{*}\left(F e^{i \arg A U}\right)
$$

where

$$
A^{*} f=\frac{c_{1} c_{2}}{(2 \pi)^{2}} \iint_{\bar{\Omega}} f\left(s_{1}, s_{2}\right) \mathrm{e}^{i\left(c_{1} x s_{1}+c_{2} y s_{2}\right)} \mathrm{d} s_{1} \mathrm{~d} s_{2}
$$

is conjugate operator with $A$.

Further we introduce to shorten records the following notations:

$$
Q=\left(s_{1}, s_{2}\right), \mathrm{d} Q=\mathrm{d} s_{1} \mathrm{~d} s_{2}, P=(x, y), \quad \mathrm{d} P=\mathrm{d} x \mathrm{~d} y .
$$

Taking into account that a set of zeros $N(A)$ consists only of zero element, and acting on both parts of (5) by operator $A$, we obtain equivalent to (5) equation with respect to function $f\left(s_{1}, s_{2}\right)$ in the space $L_{2}(G)$

$$
\alpha f=-A A^{*} f+A A^{*}\left(F e^{i \arg f}\right) \text {. }
$$

Accordingly to the introduced above notations this equation in the expanded form takes the form

$$
\begin{aligned}
& \alpha f(Q)=\mathbf{B} f \equiv-\iint_{\bar{\Omega}} K\left(Q, Q^{\prime}, \mathbf{c}\right) f\left(Q^{\prime}\right) \mathrm{d} Q^{\prime} \\
& +\iint_{\bar{\Omega}} K\left(Q, Q^{\prime}, \mathbf{c}\right) F\left(Q^{\prime}\right) \mathrm{e}^{i \arg f\left(Q^{\prime}\right)} \mathrm{d} Q^{\prime},
\end{aligned}
$$

where

$$
\begin{aligned}
& K\left(Q, Q^{\prime}, \mathbf{c}\right) \\
& =\frac{c_{1} c_{2}}{(2 \pi)^{2}} \iint_{G} \exp \left[i\left(c_{1} x\left(s_{1}^{\prime}-s_{1}\right)+c_{2} y\left(s_{2}^{\prime}-s_{2}\right)\right)\right] \mathrm{d} x \mathrm{~d} y
\end{aligned}
$$

is a kernel dependent on the form of the domain $G$. In the case of symmetric domain $G$ (8) can be simplified. In particular, if the axis $O X$ is the axis of symmetry of the domain $G$ and its upper and lower limits are described, respectively, by the functions $y= \pm \eta(x)$ at $x \in[-1,1]$, the kernel (8) is real and it has the form

$$
\begin{aligned}
& K\left(Q, Q^{\prime}, \mathbf{c}\right) \\
& =\frac{c_{1}}{2 \pi^{2}} \int_{-1}^{1} \cos \left(c_{1} x\left(s_{1}^{\prime}-s_{1}\right)\right) \frac{\sin \left(c_{2}\left(s_{2}^{\prime}-s_{2}\right) \eta(x)\right)}{\left(s_{2}^{\prime}-s_{2}\right)} \mathrm{d} x .
\end{aligned}
$$

Lemma 1. Between solutions of Equations (5) and (6) there exists bijection, that is if $U_{*}$ is the solution of (5) then $f_{*}=A U_{*}$ is the solution of (6). On the contrary, if $f_{*}$ is the solution of (6) then

$U_{*}=A^{*}\left\{-\alpha^{-1} f_{*}+\alpha^{-1} F \exp \left(i \arg \left(f_{*}\right)\right)\right\}$ is the solution of $(5)$.

Proof. Let $U_{*}$ be a solution of (5). Then $U_{*}+\alpha^{-1} A^{*} A U_{*}-\alpha^{-1} A^{*}\left(F e^{i \arg A U_{*}}\right) \equiv 0$. Acting on this equality by the operator $A$, we obtain

$A U_{*}+\alpha^{-1} A A^{*} A U_{*}-\alpha^{-1} A A^{*}\left(F e^{i \arg A U_{*}}\right) \equiv 0$. The operator acts from the space $L_{2}(G)$ into the space $L_{2}(\Omega)$, and a set of its zeros consists only of zero element. Then from the last identity follows that $A U_{*}=f_{*} \in L_{2}(\Omega)$ is a solution of the equation

$$
f_{*}+\alpha^{-1} A A^{*} f_{*}-\alpha^{-1} A A^{*}\left(F e^{i \arg f_{*}}\right)=0 \text {, that is (6). }
$$

On the contrary, let $f_{*} \in L_{2}(\Omega)$ be a solution of (6). The operator $A^{*}$ acts from the space $L_{2}^{*}(\Omega)$ into the space $L_{2}^{*}(G)$ [6], and the Hilbertian space $L_{2}^{*}$ coincides with the space $L_{2}$ [5]. From here follows, that $A^{*}$ acts from the space $L_{2}(\Omega)$ into the space $L_{2}(G)$. Taking into account that $F$ and $f_{*}$ are continuous functions, the function $F \exp \left(i \arg \left(f_{*}\right)\right)$ is quadratically integrable in the domain $\Omega$. From here follows

$$
\left\{-\alpha^{-1} f_{*}+\alpha^{-1} F \exp \left(i \arg \left(f_{*}\right)\right)\right\} \in L_{2}(\Omega) .
$$

Thus,

$$
A^{*}\left\{-\alpha^{-1} f_{*}+\alpha^{-1} F \exp \left(i \arg \left(f_{*}\right)\right)\right\}=U_{*} \in L_{2}(G)
$$

and the right part of (6) is a result of action of operator $A$ on the element $U_{*}$, that is

$$
A U_{*}=A A^{*}\left\{-\alpha^{-1} f_{*}+\alpha^{-1} F \exp \left(i \arg \left(f_{*}\right)\right)\right\}=f_{*} .
$$

Thus owing to the fact that $A U_{*}=f_{*}$, we write this equality in the form

$$
A\left\{U_{*}+\alpha^{-1} A^{*} A U_{*}-\alpha^{-1} A^{*} F \exp \left(i \arg \left(A U_{*}\right)\right)\right\}=0 .
$$

Since a set of zeros of operator $A$ consists only of 
zero element we have

$$
U_{*}=-\alpha^{-1} A^{*} A U_{*}+\alpha^{-1} A^{*} F \exp \left(i \arg \left(A U_{*}\right)\right) .
$$

So,

$$
U_{*}=A^{*}\left\{-\alpha^{-1} f_{*}+\alpha^{-1} F \exp \left(i \arg \left(f_{*}\right)\right)\right\}
$$

solves (5). Lemma is proved.

Using the general expression (8) for the kernel $K\left(Q, Q^{\prime}, \mathbf{c}\right)$ we shall consider a self-adjoint operator

$$
D f=A A^{*} f \equiv \iint_{\Omega} K\left(Q, Q^{\prime}, \mathbf{c}\right) f\left(Q^{\prime}\right) \mathrm{d} Q^{\prime}
$$

and corresponding to it quadratic form for arbitrary function $f(Q) \in L_{2}(\Omega)$ :

$$
\begin{aligned}
& (D f, f)=\iint_{\Omega} \iint_{\Omega} K\left(Q, Q^{\prime}, \mathbf{c}\right) f\left(Q^{\prime}\right) \mathrm{d} Q^{\prime} \overline{f(Q)} \mathrm{d} Q \\
& =\frac{c_{2} c_{2}}{(2 \pi)^{2}} \iint_{G}\left|\iint_{\Omega} f(Q) \exp (i \mathbf{c}(P, Q)) \mathrm{d} Q\right|^{2} \mathrm{~d} P \geq 0 .
\end{aligned}
$$

This equality to zero is achieved only as $f(Q) \equiv 0$. From here follows that the operator $D$ is nonnegative in $L_{2}(\Omega)$ [7] and, respectively, in $C(\bar{\Omega})$. Based on this property, the operator $D$ retains the nonnegative functions cone $\mathrm{K} \subset C(\bar{\Omega})$ invariant, that is $D \mathrm{~K} \subset \mathrm{K}$ [8].

Since a set of values of operator $A$ is a set of continuous functions [5], belonging to the space $L_{2}(\Omega)$, and a set of continuous functions in the domain $\bar{\Omega}$, is dense in the space $L_{2}(\Omega)$ [5], we shall investigate the solutions of (6) in the space $L_{2}(\Omega)$.

On the basis of decomplexification [7] we consider the complex space $\mathbb{C}(\bar{\Omega})$ as a direct sum

$\mathbb{C}(\bar{\Omega})=C(\bar{\Omega}) \oplus C(\bar{\Omega})$ of two real spaces of continuous functions in the domain $\bar{\Omega}$. The elements of this space have the form: $f=(u, v)^{T} \in \mathbb{C}(\bar{\Omega})$, $u=\operatorname{Re}(f) \in C(\bar{\Omega}), \quad v=\operatorname{Im}(f) \in C(\bar{\Omega})$. Norms in these spaces we shall introduce as:

$$
\begin{aligned}
\|u\|_{C(\bar{\Omega})} & =\max _{Q \in \bar{\Omega}}|u(Q)|,\|v\|_{C(\bar{\Omega})}=\max _{Q \in \bar{\Omega}}|v(Q)|, \\
\|f\|_{\mathbb{C}(\bar{\Omega})} & =\max \left(\|u\|_{C(\bar{\Omega})},\|v\|_{C(\bar{\Omega})}\right) .
\end{aligned}
$$

The Equation (6) in decomplexified space $\mathbb{C}(\bar{\Omega})$ we reduce to equivalent to it system of equations

$$
\left\{\begin{array}{l}
u=B_{1}(u, v) \equiv-\alpha^{-1} B_{11} u+\alpha^{-1} B_{12}(u, v) \\
v=B_{2}(u, v) \equiv-\alpha^{-1} B_{21} v+\alpha^{-1} B_{22}(u, v)
\end{array}\right.
$$

where

$$
\begin{gathered}
B_{11}=\iint_{\bar{\Omega}} K\left(Q, Q^{\prime}, \mathbf{c}\right) u\left(Q^{\prime}\right) \mathrm{d} Q^{\prime} \\
B_{12}=\iint_{\bar{\Omega}} F\left(Q^{\prime}\right) K\left(Q, Q^{\prime}, \mathbf{c}\right) \frac{u\left(Q^{\prime}\right)}{\sqrt{u^{2}\left(Q^{\prime}\right)+v^{2}\left(Q^{\prime}\right)}} \mathrm{d} Q^{\prime}
\end{gathered}
$$

$$
\begin{gathered}
B_{21}=\iint_{\bar{\Omega}} K\left(Q, Q^{\prime}, \mathbf{c}\right) v\left(Q^{\prime}\right) \mathrm{d} Q^{\prime}, \\
B_{22}=\iint_{\bar{\Omega}} F\left(Q^{\prime}\right) K\left(Q, Q^{\prime}, \mathbf{c}\right) \frac{v\left(Q^{\prime}\right)}{\sqrt{u^{2}\left(Q^{\prime}\right)+v^{2}\left(Q^{\prime}\right)}} \mathrm{d} Q^{\prime} .
\end{gathered}
$$

Note, $B_{11} B_{21}$ are linear integral operators and $B_{11}=B_{21}$.

Denote a closed convex set of continuous functions as $S_{M} \subset \mathbb{C}(\bar{\Omega})$ supposing that

$$
\begin{aligned}
& S_{M}=S_{M_{u}} \oplus S_{M_{v}}, S_{M_{u}}=\left\{u \in S_{M_{u}}:\|u\|_{C(\bar{\Omega})} \leq q M\right\}, \\
& S_{M_{v}}=\left\{v \in S_{M_{v}}:\|v\|_{C(\bar{\Omega})} \leq q M\right\},
\end{aligned}
$$

where

$$
\begin{aligned}
& q=\left\|\left(I+\alpha^{-1} B_{11}\right)^{-1}\right\|_{C(\bar{\Omega}) \rightarrow C(\bar{\Omega})}, \\
& M=\max _{Q \in \bar{\Omega}} \iint_{\bar{\Omega}} F\left(Q^{\prime}\right)\left|K\left(Q, Q^{\prime}, \mathbf{c}\right)\right| \mathrm{d} Q^{\prime},
\end{aligned}
$$

$I$ is a unit operator in $C(\bar{\Omega})$.

We show that onerator $\mathbf{B}=\left(B_{1}, B_{2}\right)^{T}$ determined by (13) acts in the space $\mathbb{C}(\bar{\Omega})$. At first consider $B_{1}(u, v)$. The first component of this operator, defined by (14), is a linear integral operator with the kernel $K\left(Q, Q^{\prime}, \mathbf{c}\right)$ which is continuous on both arguments. Consequently, $B_{11}: C(\bar{\Omega}) \rightarrow C(\bar{\Omega})$ is a continuous operator [9].

Show that $B_{12}: \mathbb{C}(\bar{\Omega}) \rightarrow \mathbb{C}(\bar{\Omega})$. Let $f=(u, v)^{T}$ be an arbitrary function belonging to $\mathbb{C}(\bar{\Omega})$. For

$0<c_{i}<\infty \quad(i=1,2)$ the kernel $K\left(Q, Q^{\prime}, \mathbf{c}\right)$ is a continuous function with respect to its arguments in the closed domain $\bar{\Omega} \times \bar{\Omega}$. Then according to the Cantor theorem [10] $K\left(Q, Q^{\prime}, \mathbf{c}\right)$ is a uniformly continuous function in $\bar{\Omega} \times \bar{\Omega}$. From here follows: at fixed c for any points $\left(Q_{1}, Q_{1}^{\prime}\right)$ and $\left(Q_{2}, Q_{2}^{\prime}\right)$ such that whenever $\left|\left(Q_{1}, Q_{1}^{\prime}\right)-\left(Q_{2}, Q_{2}^{\prime}\right)\right|<\delta$, then

$$
\left|K\left(Q_{1}, Q_{1}^{\prime}, \mathbf{c}\right)-K\left(Q_{2}, Q_{2}^{\prime}, \mathbf{c}\right)\right|<\frac{\varepsilon}{a} \text {, here } a=\iint_{\bar{\Omega}} F\left(Q^{\prime}\right) \mathrm{d} Q^{\prime} .
$$

On this basis we have

$$
\begin{aligned}
& \left|\tilde{u}\left(Q_{1}\right)-\tilde{u}\left(Q_{2}\right)\right| \\
& =\mid \iint_{\bar{\Omega}} F\left(Q^{\prime}\right)\left[K\left(Q_{1}, Q^{\prime}, \mathbf{c}\right)-K\left(Q_{2}, Q^{\prime}, \mathbf{c}\right)\right] \\
& \quad \frac{u\left(Q^{\prime}\right)}{\sqrt{u^{2}\left(Q^{\prime}\right)+v^{2}\left(Q^{\prime}\right)}} \mathrm{d} Q^{\prime} \mid \\
& \leq \frac{\varepsilon}{a} \iint_{\bar{\Omega}} F\left(Q^{\prime}\right) \mathrm{d} Q^{\prime}=\varepsilon
\end{aligned}
$$

since $\max _{Q^{\prime} \in \bar{\Omega}}\left|\frac{u\left(Q^{\prime}\right)}{\sqrt{u^{2}\left(Q^{\prime}\right)+v^{2}\left(Q^{\prime}\right)}}\right| \leq 1$. Thus, $\tilde{u}=B_{12}(u, v)$ 
is a continuous function and $B_{12}: \mathbb{C}(\bar{\Omega}) \rightarrow \mathbb{C}(\bar{\Omega})$. Analogously we show that $B_{22}: \mathbb{C}(\bar{\Omega}) \rightarrow \mathbb{C}(\bar{\Omega})$.

Consider corresponding to (13) linear homogeneous equation

$$
-\alpha u(Q)=\iint_{\Omega} K\left(Q, Q^{\prime}, \mathbf{c}\right) u\left(Q^{\prime}\right) \mathrm{d} Q^{\prime} .
$$

Above it is shown that the integral operator in the right part of (18) is self-adjoint and positively determined. Hence, its eigenvalues are real and nonnegative [9]. From here follows that $\alpha$ cannot be eigenvalue of (18). Then this equation has only zero solution $u(Q) \equiv 0$. Thus it is shown that necessary and sufficient condition for existence of inverse operator $\left(I+\alpha^{-1} B_{11}\right)^{-1}$ is satisfied [11]. Since $B_{21}=B_{11}$, then $\left(I+\alpha^{-1} B_{21}\right)^{-1}$ exists too. Easily to show [12] that

$$
\begin{aligned}
& \left\|\left(I+\alpha^{-1} B_{11}\right)^{-1}\right\| \\
& =\sup _{u \in L_{2}, u \neq 0} \frac{\left(\left(I+\alpha^{-1} B_{11}\right)^{-1} u,\left(I+\alpha^{-1} B_{11}\right)^{-1} u\right)}{(u, u)}=q \leq 1 .
\end{aligned}
$$

From here follows, that $\left(I+\alpha^{-1} B_{11}\right)^{-1}$ is a limited operator.

Using existence and limitations of the operators $\left(I+\alpha^{-1} B_{11}\right)^{-1}$ and $\left(I+\alpha^{-1} B_{21}\right)^{-1}$ we write (13) in the form

$$
\left\{\begin{array}{l}
u=B_{1}(u, v) \equiv \alpha^{-1}\left(I+\alpha^{-1} B_{11}\right)^{-1} B_{12}(u, v), \\
v=B_{2}(u, v) \equiv \alpha^{-1}\left(I+\alpha^{-1} B_{21}\right)^{-1} B_{22}(u, v) .
\end{array}\right.
$$

Theorem 1. The operator $\mathbf{B}=\left(B_{1}, B_{2}\right)^{T}$ determined by (19) maps a closed convex set $S_{M}$ of the Banach space $\mathbb{C}(\bar{\Omega})$ in itself and it is completely continuous.

Proof. Before it was shown that $\mathbf{B}: \mathbb{C}(\bar{\Omega}) \rightarrow \mathbb{C}(\bar{\Omega})$.

To prove the property of complete continuity of the operator $\mathbf{B}=\left(B_{1}, B_{2}\right)^{T}$ it is necessary to prove its compactness and continuity [7]. We consider each of operators $B_{1}(u, v)$ and $B_{2}(u, v)$ in a system of Equations (19) as the product of linear limited (continuous) and nonlinear operators. Since $\left(I+\alpha^{-1} B_{11}\right)^{-1},\left(I+\alpha^{-1} B_{21}\right)^{-1}$ are limited operators, then for complete continuity of the operator $\mathbf{B}=\left(B_{1}, B_{2}\right)^{T}$ it is sufficient to show complete continuity of operators $B_{12}(u, v), B_{22}(u, v)$. We shall show it on the example $B_{12}(u, v)$.

Let $f_{1}=\left(u_{1}, v_{1}\right)^{T}$ and $f_{2}=\left(u_{2}, v_{2}\right)^{T}$ be arbitrary functions belonging to $S_{M}$, and $u_{1} \neq 0$ or $v_{1} \neq 0$. It is necessary to show, that $\left\|B_{12} f_{1}-B_{12} f_{2}\right\|_{C(\bar{\Omega})} \rightarrow 0$ as $\left\|f_{1}-f_{2}\right\|_{\mathbb{C}(\bar{\Omega})} \rightarrow 0$. Let us assume $u_{2}=u_{1}+\Delta u$, $v_{2}=v_{1}+\Delta v$ Taking into account these equalities we obtain ${ }^{2}$

${ }^{2}$ Further for reduction of notations the dependence of functions $u_{1}, u_{2}$, $\Delta u, \Delta v$ on the variable $Q$ is omitted in (22) and (23) .
$\frac{u_{2}}{\sqrt{u_{2}^{2}+v_{2}^{2}}}=\frac{u_{1}+\Delta u}{\sqrt{u_{1}^{2}+v_{1}^{2}} \sqrt{1+\frac{2 u_{1} \Delta u+2 v_{1} \Delta v+\Delta u^{2}+\Delta v^{2}}{u_{1}^{2}+v_{1}^{2}}}}$.

At $\|\Delta u\|_{C(\bar{\Omega})} \rightarrow 0,\|\Delta v\|_{C(\bar{\Omega})} \rightarrow 0$ we have:

$$
\lim _{\|\Delta u\| \rightarrow 0,}\left\|\frac{u_{1}}{\Delta \Delta v \| \rightarrow \rightarrow v_{1}^{2}}-\frac{u_{2}}{\sqrt{u_{2}^{2}+v_{2}^{2}}}\right\|_{\mathbb{C}(\bar{\Omega})}
$$$$
\leq \lim _{\substack{\Delta \Delta\|\rightarrow 0,0 \\ \Delta v\| \rightarrow 0}} \max _{Q \in \Omega}\left\{\frac{u_{1}}{\sqrt{u_{1}^{2}+v_{1}^{2}}}\right.
$$

$$
\cdot\left(1-\frac{1}{\sqrt{1+\frac{2 u_{1} \Delta u+2 v_{1} \Delta v+\Delta u^{2}+\Delta v^{2}}{u_{1}^{2}+v_{1}^{2}}}}\right)
$$

$$
+\left|\frac{\Delta u}{\sqrt{u_{1}^{2}+v_{1}^{2}} \sqrt{1+\frac{2 u_{1} \Delta u+2 v_{1} \Delta v+\Delta u^{2}+\Delta v^{2}}{u_{1}^{2}+v_{1}^{2}}}}\right|=0 \text {, }
$$

since $\lim _{\substack{\Delta u \\ \Delta v\|\rightarrow 0,0 \\ \Delta v\| \rightarrow=\Omega}}\left|\left(\sqrt{1+\frac{2 u_{1} \Delta u+2 v_{1} \Delta v+\Delta u^{2}+\Delta v^{2}}{u_{1}^{2}+v_{1}^{2}}}\right)\right|=1$.

Analogously we obtain

$$
\lim _{\substack{\Delta u \\ \Delta \nu \|, 0,0}} \max _{\substack{\Delta \\ \| \in \Omega}}\left|\frac{v_{1}}{\sqrt{u_{1}^{2}+v_{1}^{2}}}-\frac{v_{2}}{\sqrt{u_{2}^{2}+v_{2}^{2}}}\right|=0 .
$$

Thus, from equalities (20) and (21) follows

$$
\begin{aligned}
& \lim _{\substack{\Delta u \rightarrow \rightarrow 0 \\
\Delta \nu \|, 0}} \mid B_{12}\left(u_{1}, v_{1}\right)-B_{12}\left(u_{2}, v_{2}\right) \|_{\mathbb{C}(\bar{\Omega})}= \\
& =\lim _{\substack{\Delta \Delta\|\rightarrow 0,0 \\
\Delta v\| \rightarrow 0}} \max _{Q \in \Omega} \mid \iint_{\bar{\Omega}} F\left(Q^{\prime}\right) K\left(Q, Q^{\prime}, \mathbf{c}\right) \times \\
& \times\left\{\frac{u_{1}\left(Q^{\prime}\right)}{\sqrt{u_{1}^{2}\left(Q^{\prime}\right)+v_{1}^{2}\left(Q^{\prime}\right)}}-\frac{u_{2}\left(Q^{\prime}\right)}{\sqrt{u_{2}^{2}\left(Q^{\prime}\right)+v_{2}^{2}\left(Q^{\prime}\right)}}\right\} \mathrm{d} Q^{\prime} \mid=0
\end{aligned}
$$

Analogously

$$
\lim _{\substack{\Delta \Delta\left\|_{C(\bar{\Omega}) \rightarrow 0,}\\\right\| \Delta \|_{C(\bar{\Omega})} \rightarrow 0}}\left\|B_{22}\left(u_{1}, v_{1}\right)-B_{22}\left(u_{2}, v_{2}\right)\right\|_{\mathbb{C}(\bar{\Omega})}=0 .
$$

Therefore, $\mathbf{B}=\left(B_{1}, B_{2}\right)^{T}$ is a continuous operator from $\mathbb{C}(\bar{\Omega})$ into $\mathbb{C}(\bar{\Omega})$.

Show that a set of functions $S_{w}=B_{12} S_{M}$ satisfies conditions of the Arzela Theorem [5], that is we show 
that functions of a set $S_{w}$, are uniformly bounded and equapotentially continuous. Let $w=B_{12}(u, v)$, where $f=(u, v)^{T}$ is an arbitrary function of a set $S_{M}$. Then for $\left|\left(Q_{1}, Q^{\prime}\right)-\left(Q_{2}, Q^{\prime}\right)\right|<\delta$ analogously with (17) we have

$$
\left|w\left(Q_{1}\right)-w\left(Q_{2}\right)\right| \leq \frac{\varepsilon}{a} \iint_{\bar{\Omega}} F\left(Q^{\prime}\right) \mathrm{d} Q^{\prime} \leq \varepsilon .
$$

Thus the functions of a set $S_{w}=B_{12} S_{M}$ are equapotentially continuous.

Uniform boundedness of a set $S_{w}=B_{12} S_{M}$ follows from inequality

$$
\begin{aligned}
& \|w\|_{\mathbb{C}(\bar{\Omega})} \\
& =\max _{Q \in \bar{\Omega}}\left|\iint_{\bar{\Omega}} F\left(Q^{\prime}\right) K\left(Q, Q^{\prime}, \mathbf{c}\right) \frac{u\left(Q^{\prime}\right)}{\sqrt{u^{2}\left(Q^{\prime}\right)+v^{2}\left(Q^{\prime}\right)}} \mathrm{d} Q^{\prime}\right| \\
& \leq \max _{Q \in \bar{\Omega}} \iint_{\bar{\Omega}} F\left(Q^{\prime}\right)\left|K\left(Q, Q^{\prime}, \mathbf{c}\right)\right| \frac{u\left(Q^{\prime}\right)}{\sqrt{u^{2}\left(Q^{\prime}\right)+v^{2}\left(Q^{\prime}\right)}} \mid \mathrm{d} Q^{\prime} \leq M,
\end{aligned}
$$

where $f=(u, v)^{T}$ is an arbitrary function of a set $S_{M}$.

Thus the operator $B_{1}$ is completely continuous in the first equation of system (19). Complete continuity of operator $B_{2}$ is proved analogously.

Let $f=(u, v)^{T}$ be an arbitrary function of a set $S_{M}$ and $(g, h)^{T}=\mathbf{B}(u, v)^{T}$. Show that the function $(g, h)^{T}$ belongs to a set $S_{M}$. Using the inequalities

$$
\begin{gathered}
\|A x\| \leq\|A\|\|x\| \text { and }\left\|\left(I+\alpha^{-1} B_{11}\right)^{-1}\right\|=q \leq 1 \text { we have } \\
\|g(Q)\|_{C(\bar{\Omega})}=\left\|B_{1}(u, v)\right\|_{C(\bar{\Omega}) \rightarrow C(\bar{\Omega})} \leq \\
\leq\left\|\left(I+\alpha^{-1} B_{11}\right)^{-1}\right\|_{C(\bar{\Omega}) \rightarrow C(\bar{\Omega})}\|w\|_{C(\bar{\Omega})} \leq q M .
\end{gathered}
$$

Analogously we obtain:

$$
\begin{aligned}
& \|h(Q)\|_{C(\bar{\Omega})}\left\|B_{2}(u, v)\right\|_{C(\bar{\Omega}) \rightarrow C(\bar{\Omega})} \leq \\
& \leq\left\|\left(I+\alpha^{-1} B_{21}\right)^{-1}\right\|_{C(\bar{\Omega}) \rightarrow C(\bar{\Omega})}\|\omega\|_{C(\bar{\Omega})} \leq q M .
\end{aligned}
$$

From these inequalities follows that $\mathbf{B} S_{M} \subset S_{M}$. So, the operator $\mathbf{B}=\left(B_{1}, B_{2}\right)^{T}$ is completely continuous mapping the closed convex set $S_{M} \subset \mathbb{C}(\bar{\Omega})$ into itself.

The theorem is proved.

As a corollary of Theorem 1 satisfaction of the Schauder principle conditions [7] in accordance to which the operator $\mathbf{B}=\left(B_{1}, B_{2}\right)^{T}$ has a fixed point $f_{*}=\left(u_{*}, v_{*}\right)^{T}$, belonging to a set $S_{M}$, follows. This point solves (13) and, respectively, (6). Substituting $f_{*}=\left(u_{*}, v_{*}\right)^{T}$ into (10), we obtain the solution of $(5)$, which is a stationary point of functional (4).

Concerning the synthesis problems of linear radiator for the case of one-dimensional domains $\bar{\Omega}$ the solu- tions of system of equations similar to (13) are investigated, in particular, in [13]. The obtained there results show that nonuniquness and branching of solutions, dependent on the size of physical parameters of the problem are characteristic for special case of equations of type (13) (when variables are separated). The results [13] can not be transferred directly to two-dimensional nonlinear integral equations of type (7). Here unlike the branching points [13] there exist branching lines of solutions, and the problem of finding the branching lines is not enough investigated nonlinear two-parameter spectral problem.

\section{Equations of a Set of Branching Points}

In the case when the kernel of (7) determined by (8) is real, (7) in a space of real continuous functions $C(\bar{\Omega})$ has the form

$$
\begin{aligned}
& \alpha f(Q)+\iint_{\bar{\Omega}} K\left(Q, Q^{\prime}, \mathbf{c}\right) f\left(Q^{\prime}\right) \mathrm{d} Q^{\prime} \\
& =\iint_{\bar{\Omega}} F\left(Q^{\prime}\right) K\left(Q, Q^{\prime}, \mathbf{c}\right) \operatorname{sign}\left(f\left(Q^{\prime}\right)\right) \mathrm{d} Q^{\prime} .
\end{aligned}
$$

Assuming in (23) $\operatorname{sign} f\left(Q^{\prime}\right) \equiv 1$ we obtain the second kind Fredholm integral linear equation with symmetric and even kernel

$$
f(Q)+\alpha^{-1} \iint_{\bar{\Omega}} K\left(Q, Q^{\prime}, \mathbf{c}\right) f\left(Q^{\prime}\right) \mathrm{d} Q^{\prime}=\mathrm{F}(Q) .
$$

Here the right part

$$
\mathrm{F}(Q)=\alpha^{-1} \iint_{\bar{\Omega}} F\left(Q^{\prime}\right) K\left(Q, Q^{\prime}, \mathbf{c}\right) \mathrm{d} Q^{\prime}
$$

is a nonnegative function. It was shown that corresponding to (24) homogeneous Equation (18) has only zero solution. From here follows that (24) has unique solution $f_{0}(Q)$, belonging to the space $C(\bar{\Omega})$ and $\operatorname{sign} f_{0}(Q) \equiv 1$, that is solution $f_{0}(Q)$ is nonnegative.

Further we shall call the solution $f_{0}(Q, \mathbf{c})$ as initial solution of (7). Corresponding to it solution

$$
\left\{\begin{array}{l}
u_{0}(Q, \mathbf{c})=f_{0}(Q, \mathbf{c}), \\
v_{0}(Q, \mathbf{c})=0
\end{array}\right.
$$

we shall call as initial solution of a system of Equations (13).

To find the branching lines and complex solutions of (7), which branch-off from the real solution $f_{0}(Q, \mathbf{c})$ we consider the problem on finding such a set of values of parameters $\mathbf{c}^{(0)}=\left(c_{1}^{(0)}, c_{2}^{(0)}\right)$ and all distinct from $f_{0}(Q, \mathbf{c})$ solutions of (13), which satisfy the conditions

$$
\left\{\begin{array}{l}
\max _{Q \in \bar{\Omega}}\left|u(Q, \mathbf{c})-f\left(Q, \mathbf{c}^{(0)}\right)\right| \rightarrow 0, \\
\max _{Q \in \bar{\Omega}}|v(Q, \mathbf{c})| \rightarrow 0
\end{array}\right.
$$


as $\left|\mathbf{c}-\mathbf{c}^{(0)}\right| \rightarrow 0$. Conditions (25) mean that it is necessary to find small continuous solutions in $\bar{\Omega}$

$$
w(Q, \mathbf{c})=u(Q, \mathbf{c})-f_{0}\left(Q, \mathbf{c}^{(0)}\right), \omega(Q, \mathbf{c})=v(Q, \mathbf{c}),
$$

converging uniformly to zero as $\mathbf{c} \rightarrow \mathbf{c}^{(0)}$. In addition, it is necessary also to take into account the direction of convergence of vector $\mathbf{c}$ to $\mathbf{c}^{(0)}$.

Put

$$
c_{1}=c_{1}^{(0)}+\mu, \quad c_{2}=c_{2}^{(0)}+v
$$

and we shall find the desired solution in the form

$$
\begin{aligned}
& u(Q, \mathbf{c})=f_{0}\left(Q, \mathbf{c}^{(0)}\right)+w(Q, \mu, v) \\
& v(Q, \mathbf{c})=\omega(Q, \mu, v) .
\end{aligned}
$$

Further we omit the dependence of functions $w(Q, \mu, v)$ and $\omega(Q, \mu, v)$ on parameters $\mu$ and $v$ for reduction of notations.

Present some properties of integrands in (13). They are continuous functions of arguments. We substitute (26) and (27) in (13). Then integrands expand in the uniformly convergence power series with respect to the functional arguments $w, \omega$ and numerical parameters $\mu, v$ in the neighborhood of point $\left(\mathbf{c}^{(0)}, f_{0}\left(Q, \mathbf{c}^{(0)}\right), 0\right)$ :

$$
\begin{aligned}
& K\left(Q, Q^{\prime}, \mathbf{c}\right) \times\left(-u\left(Q^{\prime}\right)+F\left(Q^{\prime}\right) \frac{u\left(Q^{\prime}\right)}{\sqrt{u^{2}\left(Q^{\prime}\right)+v^{2}\left(Q^{\prime}\right)}}\right) \\
& =\sum_{m+n+p+q \geq 0} A_{m n p q}\left(Q, Q^{\prime}, \mathbf{c}^{(0)}\right) w^{m}\left(Q^{\prime}\right) \omega^{n}\left(Q^{\prime}\right) \mu^{p} v^{q}, \\
& K\left(Q, Q^{\prime}, \mathbf{c}\right) \times\left(-v\left(Q^{\prime}\right)+F\left(Q^{\prime}\right) \frac{v\left(Q^{\prime}\right)}{\sqrt{u^{2}\left(Q^{\prime}\right)+v^{2}\left(Q^{\prime}\right)}}\right) \\
& =\sum_{m+n+p+q \geq 1} B_{m n p q}\left(Q, Q^{\prime}, \mathbf{c}^{(0)}\right) w^{m}\left(Q^{\prime}\right) \omega^{n}\left(Q^{\prime}\right) \mu^{p} v^{q} .
\end{aligned}
$$

Here $A_{\text {mnpq }}\left(Q, Q^{\prime}, \mathbf{c}^{(0)}\right), B_{\text {mnpq }}\left(Q, Q^{\prime}, \mathbf{c}^{(0)}\right)$ are coefficients of expansion continuously dependent on arguments. Substituting (28) in (13) and considering $f_{0}\left(Q^{\prime}, \mathbf{c}^{(0)}\right)$ as a solution of this system, we obtain a system of nonlinear equations with respect to small solutions $w$ and $\omega$ :

$$
\begin{aligned}
& \alpha w(Q)+\iint_{\bar{\Omega}} K\left(Q, Q^{\prime}, \mathbf{c}^{(0)}\right) w\left(Q^{\prime}\right) \mathrm{d} Q^{\prime} \\
& =\frac{c}{2 \pi} a_{10}\left(Q, \mathbf{c}^{(0)}\right) \mu+a_{01}\left(Q, \mathbf{c}^{(0)}\right) v+ \\
& +\sum_{m+n+p+q \geq 2} \mu^{p} v^{q} \iint_{\Omega} A_{m n p q}\left(Q, Q^{\prime}, \mathbf{c}^{(0)}\right) w^{m}\left(Q^{\prime}\right) \omega^{n}\left(Q^{\prime}\right) \mathrm{d} Q^{\prime},
\end{aligned}
$$

$$
\begin{aligned}
& \alpha \omega(Q)-\iint_{\bar{\Omega}}\left(F\left(Q^{\prime}\right)-1\right) K\left(Q, Q^{\prime}, \mathbf{c}^{(0)}\right) \frac{\omega\left(Q^{\prime}\right)}{f_{0}\left(Q^{\prime}, \mathbf{c}^{(0)}\right)} \mathrm{d} Q^{\prime}= \\
& =\sum_{m+n+p+q \geq 2} \mu^{p} v^{q} \iint_{\Omega} B_{m} m p q\left(Q, Q^{\prime}, \mathbf{c}^{(0)}\right) w^{m}\left(Q^{\prime}\right) \omega^{n}\left(Q^{\prime}\right) \mathrm{d} Q^{\prime},
\end{aligned}
$$

where

$$
\begin{aligned}
& a_{10}\left(Q, \mathbf{c}^{(0)}\right)=\iint_{\Omega} A_{0010}\left(Q, Q^{\prime}, \mathbf{c}^{(0)}\right) \mathrm{d} Q^{\prime}, \\
& a_{01}\left(Q, \mathbf{c}^{(0)}\right)=\iint_{\Omega} A_{0001}\left(Q, Q^{\prime}, \mathbf{c}^{(0)}\right) \mathrm{d} Q^{\prime} .
\end{aligned}
$$

Extracting in a system of Equations (29), (30) the linear members for $w$ and $\omega$, to find a set of branching points of the solution $f_{0}\left(Q^{\prime}, \mathbf{c}\right)$ we have an integrated system of linear integral equations

$$
w(Q)=-\alpha^{-1} \iint_{\bar{\Omega}} K\left(Q, Q^{\prime}, \mathbf{c}\right) w\left(Q^{\prime}\right) \mathrm{d} Q^{\prime},
$$

$$
\begin{aligned}
& \omega(Q) \\
& =\alpha^{-1} \iint_{\bar{\Omega}}\left(F\left(Q^{\prime}\right)-f_{0}\left(Q^{\prime}, \mathbf{c}\right)\right) K\left(Q, Q^{\prime}, \mathbf{c}\right) \frac{\omega\left(Q^{\prime}\right)}{f_{0}\left(Q^{\prime}, \mathbf{c}\right)} \mathrm{d} Q^{\prime} .
\end{aligned}
$$

We shall call a set of values of parameter $\mathbf{c}^{(0)}=\left\{c_{1}^{(0)}, c_{2}^{(0)}\right\}$, at which the system of linear homogeneous Equations (31), (32) has distinct from zero solutions as a set of points of possible branching of initial solution $f_{0}\left(Q^{\prime}, \mathbf{c}\right)$. Note that the system (31), (32) is noncoherent relatively the functions $w$ and $\omega$. Previously it has been shown that equation of type (31) has only a zero solution. Therefore the problem of finding a set of branching points under condition $f_{0}\left(Q^{\prime}, \mathbf{c}\right)>0$, is reduced to equation

$$
\begin{aligned}
& \varphi(Q)=T(\mathbf{c}) \varphi \\
& \equiv \alpha^{-1} \iint_{\bar{\Omega}}\left(F\left(Q^{\prime}\right)-f_{0}\left(Q^{\prime}, \mathbf{c}\right)\right) K\left(Q, Q^{\prime}, \mathbf{c}\right) \frac{\varphi\left(Q^{\prime}\right)}{f_{0}\left(Q^{\prime}, \mathbf{c}\right)} \mathrm{d} Q^{\prime},
\end{aligned}
$$

that is to a nonlinear two-dimensional spectral problem $[14,15]$. The eigenvalues of this equation form a set of points of possible branching of solutions of a system of nonlinear Equations (29), (30). Corresponding eigenfunctions are used to construct the branching-off solutions of equations [16].

Note, that construction and justification of convergence of numerical algorithms to solve the nonlinear two-parametric spectral problem on eigenvalues (33) it is necessary to solve the corresponding auxiliary one-parameter nonlinear spectral problem [14]. In this connection at first we consider two-parameter nonlinear spectral problem on the general (operational) level in the Banach spaces. 


\section{Two-Dimensional Nonlinear Spectral Problem}

\subsection{Statement of the Problem. Existence Conditions of Descrete Spectrum of Operator-Function}

Note that different approaches are used to discretization of the original problems $[7,14]$ at construction of the numerical algorithms to find the solutions of various types of nonlinear spectral problems. That is to say that original problems in the Banach functional spaces $E$ are replaced by the corresponding problems in the finite-dimensional spaces $E_{n}(n \in \mathbb{N})$. In addition the question of convergence of approximate solutions of discretization problems to exact solutions of initial problems, whenever $\operatorname{dim} E_{n} \rightarrow \infty, n \rightarrow \infty$ is important, since the input and approximated equations are considered in various spaces.

Let $E$ be a complex Banach space, $\lambda=\left(\lambda_{1}, \lambda_{2}\right)$ be a vector parameter belonging to the domain

$\Lambda=\Lambda_{1} \times \Lambda_{2}$ (open connected set) of the complex space $\mathbb{C}^{2}=\mathbb{C} \times \mathbb{C}$. Here $\lambda_{i} \in \Lambda_{i} \subset \mathbb{C}$,

$\Lambda_{i}=\left\{\lambda_{i} \in \Lambda_{i}:\left|\lambda_{i}\right|<r_{\lambda}\right\}(i=1,2), r_{\lambda}$ be some real constant. Consider the operator-function

$\mathrm{A}(\cdot, \cdot): \Lambda \rightarrow \mathrm{L}(E, E)$, which to each $\lambda=\left(\lambda_{1}, \lambda_{2}\right) \in \Lambda$ assigns the operator $\mathrm{A}\left(\lambda_{1}, \lambda_{2}\right) \in \mathrm{L}(E, E)$, where

$\mathrm{L}(E, E)$ is the space of bounded linear operators [7].

Let us consider the nonlinear two-parameter spectral problem of the form

$$
\mathrm{A}\left(\lambda_{1}, \lambda_{2}\right) x=0
$$

in which it is necessary to find the eigenvalues

$\lambda=\left(\lambda_{1}^{(0)}, \lambda_{2}^{(0)}\right) \in \Lambda$ and corresponding to them eigenvectors $x^{(0)} \in E\left(x^{(0)} \neq 0\right)$ such that $\mathrm{A}\left(\lambda_{1}^{(0)}, \lambda_{2}^{(0)}\right) x^{(0)}=0$.

In particular, in view of (33), the operator-function $\mathrm{A}\left(\lambda_{1}, \lambda_{2}\right)$ is represented as

$$
\mathrm{A}\left(\lambda_{1}, \lambda_{2}\right) \equiv\left(T\left(\lambda_{1}, \lambda_{2}\right)-I\right): \Lambda \rightarrow \mathrm{L}(E, E) .
$$

Here $T\left(\lambda_{1}, \lambda_{2}\right)$ is a linear completely continuous operator acting in the Banach space $E=C(\bar{\Omega})$ and analytically dependent on two-dimensional parameter $\left(\lambda_{1}, \lambda_{2}\right), I$ is a unique operator in $E$.

Let the Banach spaces $E, E_{n}(n=1,2, \cdots)$ and also the system $\mathrm{P}=\left(p_{n}\right)_{n \in \mathbb{N}}$ of linear bounded operators $p_{n}: E \rightarrow E_{n}$ such that

$$
\left\|p_{n} x\right\|_{E_{n}} \rightarrow\|x\|_{E}, n \in \mathbb{N}, \forall x \in E
$$

be given. Operators $p_{n}$ are called conjunctive operators $[7,14]$. From the principle of uniform boundedness [14] for $p_{n}$ the inequality follows

$$
\left\|p_{n}\right\| \leq \text { const } \quad(n \in \mathbb{N}) .
$$

Let in every space $E_{n}$ the element $x_{n}$ be selected. Writing these elements in order to increase the numbers we shall form the sequence $\left\{x_{n}\right\}$.

Using either approach to discretization of original problem the operator-function $\mathrm{A}(\cdot, \cdot): \Lambda \rightarrow \mathrm{L}(E, E)$ is approximated, respectively, by the approximate operator-functions $\mathrm{A}_{\mathrm{n}}(\cdot, \cdot): \Lambda \rightarrow \mathrm{L}\left(E_{n}, E_{n}\right), n \in \mathbb{N}$. As a result, at each $\lambda=\left(\lambda_{1}, \lambda_{2}\right) \in \Lambda$ we obtain a sequence of operators $\mathrm{A}_{n} \in \mathrm{L}\left(E_{n}, E_{n}\right)$ which convergences to operator $\mathrm{A} \in \mathrm{L}(E, E)$ at satisfaction of the corresponding conditions.

Definition of various types of convergence of operators $A_{n}$ to $A$ is given, in particular, in [14].

Discretization of original problem (33), choice of the spaces $E_{n}$ and determination of operators $p_{n}: E \rightarrow E_{n}$ are realized in various ways. In particular, if the operator-function is described by (35) and $E$ is the separable (infinite-dimensional) Hilbertian space, one of the approaches to discretization (34) consists in the following. Consider an arbitrary complete orthonormal in $E$ system of functions $\left\{x_{k}\right\}_{k=1}^{\infty}$. Each element $x \in E$ can be presented as series $x=\sum_{k=1}^{\infty} c_{k} x_{k}$ where $c_{k}=\left(x, x_{k}\right)$ is the Fourier coefficient of element $x$. If $T\left(\lambda_{1}, \lambda_{2}\right)$ is a linear continuous operator, acting in the separable Hilbertian space it admits matrix representation [9]:

$$
T_{M}\left(\lambda_{1}, \lambda_{2}\right)=\left(t_{j k}\left(\lambda_{1}, \lambda_{2}\right)\right)_{j, k=1}^{\infty},
$$

where $t_{j k}\left(\lambda_{1}, \lambda_{2}\right)=\left(T\left(\lambda_{1}, \lambda_{2}\right) x_{k}, x_{j}\right)$. In addition a sequence of the Fourier coefficients of element $y=T\left(\lambda_{1}, \lambda_{2}\right) x$ is obtained from a sequence of the Fourier coefficients of element $x$ as a result of multiplication of the matrix $T_{M}\left(\lambda_{1}, \lambda_{2}\right)$ by coefficients of element $x$.

Using the matrix representation of operator $T_{M}\left(\lambda_{1}, \lambda_{2}\right)$ the spectral problem (34) is formulated as

$$
\mathrm{A}_{M}\left(\lambda_{1}, \lambda_{2}\right) x \equiv\left(T_{M}\left(\lambda_{1}, \lambda_{2}\right)-I_{M}\right) x=0,
$$

where $I_{M}$ is a unit matrix in the space of sequences $l_{2}$.Thus, the operators $T(\lambda)$ and $T_{M}(\lambda)$ are equivalent in the sense that they to the same element $x \in E$ assign one and the same element $y \in E$. But we obtain the Fourier coefficients of element $y=(\lambda) x$ as a result of action of the operator $T_{M}(\lambda)$ on the element $x$. Obviously, that eigenvalues of these operators coincide, that is the spectral problems (34) and (38) are equivalent. In this case we nut that the finite dimensional snaces $F$ are generated by the bases $\left\{x_{k}\right\}_{k=1}^{n}(n \in \mathbb{N})$ and to each element $x \in E$ the operators $p_{n}: E \rightarrow E_{n}$ assign the element $x=\sum_{k=1}^{n} c_{k} x_{k}$ where $c_{k}=\left(x, x_{k}\right)$. As a result, operator $T_{M_{n}}(\lambda)$ approximated to $T_{M}(\lambda)$ is described 
by the finite-dimensional matrix-function

$$
T_{M_{n}}\left(\lambda_{1}, \lambda_{2}\right)=\left(t_{j k}\left(\lambda_{1}, \lambda_{2}\right)\right)_{j, k=1}^{n} .
$$

Apply other methods of discretization to (34), in particular, the quadrature (cubature) processes for the case of homogeneous integral equations and change of derivates by their difference analogues in differential equations. We obtain approximate problems to find approximate the eigenvalues and eigenvectors of matrix operator-functions in the form

$$
\mathrm{A}_{n}\left(\lambda_{1}, \lambda_{2}\right) x_{n}=0, \quad n \in \mathbb{N} .
$$

Moreover, the problem of determination the eigenvalues is reduced to finding the roots of the determinant of $n$-th order that is the roots of equation

$$
\begin{aligned}
& \Psi_{n}\left(\lambda_{1}, \lambda_{2}\right) \\
& \equiv \operatorname{det}\left(\begin{array}{cccc}
a_{11}\left(\lambda_{1}, \lambda_{2}\right) & a_{12}\left(\lambda_{1}, \lambda_{2}\right) & \cdots & a_{1 n}\left(\lambda_{1}, \lambda_{2}\right) \\
a_{21}\left(\lambda_{1}, \lambda_{2}\right) & a_{22}\left(\lambda_{1}, \lambda_{2}\right) & \cdots & a_{2 n}\left(\lambda_{1}, \lambda_{2}\right) \\
\cdots \ldots \ldots \ldots . . . & \ldots \ldots \ldots \ldots . . . & \cdots & \ldots \\
a_{n 1}\left(\lambda_{1}, \lambda_{2}\right) & a_{n 2}\left(\lambda_{1}, \lambda_{2}\right) & \cdots & a_{n n}\left(\lambda_{1}, \lambda_{2}\right)
\end{array}\right)=0 \\
& (n \in \mathbb{N})
\end{aligned}
$$

If $A_{n}\left(\lambda_{1}, \lambda_{2}\right)$ has form of (35), then

$$
a_{j, k}\left(\lambda_{1}, \lambda_{2}\right)= \begin{cases}t_{j, k}\left(\lambda_{1}, \lambda_{2}\right), & j \neq k, \\ t_{j, k}\left(\lambda_{1}, \lambda_{2}\right)-1, & j=k .\end{cases}
$$

Note if the coefficients $a_{i j}\left(\lambda_{1}, \lambda_{2}\right)$ are continuously differentiable functions of arguments, the partial derivatives $\partial \Psi_{n}\left(\lambda_{1}, \lambda_{2}\right) / \partial \lambda_{j} \quad(j=1,2)$ are determined by the rules of determinant derivation [17].

Consider the auxiliary nonlinear one-parameter spectral problem, necessary later on, as a special case of (34). Assume that variable $\lambda_{2}$ in the operator-function $\mathrm{A}\left(\lambda_{1}, \lambda_{2}\right)$ is expressed by some one-valued differentiated function $\lambda_{2}=z\left(\lambda_{1}\right)$ mapping the subdomain $\Lambda_{1, \beta} \subset \Lambda_{1}$ into some subdomain $\Lambda_{2, \beta} \subset \Lambda_{2}$. In the simplest case we put $\lambda_{2}=\beta \lambda_{1}$ ( $\beta$ is some real parameter). Introduce the operator-function

$\mathrm{A}_{\beta}\left(\lambda_{1}\right) \equiv \mathrm{A}\left(\lambda_{1}, z\left(\lambda_{1}\right)\right)$ for $\lambda_{1} \in \Lambda_{1, \beta}$, which is reduction of the operator-function $\mathrm{A}\left(\lambda_{1}, \lambda_{2}\right)$. We shall consider one-dimensional spectral problem

$$
\mathrm{A}_{\beta}\left(\lambda_{1}\right) x=0
$$

in which we assign to each value $\lambda=\left(\lambda_{1}, z\left(\lambda_{1}\right)\right) \in \Lambda$ the operator $\mathrm{A}_{\beta}\left(\lambda_{1}, z\left(\lambda_{1}\right)\right) \in \mathrm{L}(E, E)$. Analogously to (40) we consider a approximate sequence of discrete problem of (42)

$$
\mathrm{A}_{\beta, n}\left(\lambda_{1}, z\left(\lambda_{1}\right)\right) x_{n}=0, \quad n \in \mathbb{N}
$$

Denote the spectrum of operator-function $\mathrm{A}_{\beta}\left(\lambda_{1}\right)$ as $s\left(\mathrm{~A}_{\beta}\right)$. Assume that $s\left(\mathrm{~A}_{\beta}\right) \neq \Lambda_{1, \beta}$.
For the spectrum $s($ A) of the problem (34) the following theorem is valid.

Theorem 2. Let the following conditions be satisfied:

1) operator-function $\mathrm{A}(\cdot, \cdot): \Lambda \rightarrow \mathrm{L}(E, E)$ is holomorphic, and $s(\mathrm{~A}) \neq \Lambda$;

2) operator-functions $\mathrm{A}_{\mathrm{n}}(\cdot, \cdot): \Lambda \rightarrow \mathrm{L}\left(E_{n}, E_{n}\right)$ are holomorphic and for any closed bounded set $\Lambda_{0} \subset \Lambda$ the following inequality

$\max _{\lambda \in \Lambda_{0}}\left\|\mathrm{~A}_{n}\left(\lambda_{1}, \lambda_{2}\right)\right\| \leq c\left(\Lambda_{0}\right)=\mathrm{const} \quad(n \in \mathbb{N})$ is valid;

3) operators $\mathrm{A}\left(\lambda_{1}, \lambda_{2}\right) \in \mathrm{L}(E, E)$,

$\mathrm{A}_{n}\left(\lambda_{1}, \lambda_{2}\right) \in \mathrm{L}\left(E_{n}, E_{n}\right) \quad(n \in \mathbb{N})$ are the Fredholm op erators with zero index for any $\lambda=\left(\lambda_{1}, \lambda_{2}\right) \in \Lambda$;

4) spectrum $s\left(A_{\beta}\right) \neq \Lambda_{1, \beta}$ and a sequence of functions $\Psi_{n}\left(\lambda_{1}, \lambda_{2}\right)$ are differentiable in the domain $\Lambda$;

5) $\mathrm{A}_{n}(\lambda) \rightarrow \mathrm{A}(\lambda)$ is stable for any $\lambda \in r(\mathrm{~A})=\Lambda \backslash s(\mathrm{~A})$.

Then the following statements are true:

1) every point of spectrum $\lambda_{1}^{(0)} \in S\left(\mathrm{~A}_{\beta}\right)$ is isolated, it is eigenvalue of the operator $\mathrm{A}_{\beta}\left(\lambda_{1}\right) \equiv \mathrm{A}\left(\lambda_{1}, z\left(\lambda_{1}\right)\right)$, the finite-dimensional eigensubspace $N\left(\mathrm{~A}\left(\lambda_{1}^{(0)}\right)\right)$ and the finite-dimensional root subspace correspond to it;

2) for each $\lambda_{1}^{(0)} \in S\left(\mathrm{~A}_{\beta}\right)$ there exists a sequence $\left\{\lambda_{1, n}^{(0)}\right\}$ from $\lambda_{1, n}^{(0)} \in S\left(\mathrm{~A}_{\beta, n}\right) \quad\left(n>n_{0}\right)$, such that $\lambda_{1, n}^{(0)} \rightarrow \lambda_{1}^{0}$

3) each point $\lambda^{(0)}=\left(\lambda_{1}^{(0)}, z\left(\lambda_{1}^{(0)}\right)\right) \in \Lambda$ is a spectrum point of the operator-function $A\left(\lambda_{1}, \lambda_{2}\right)$;

4) if in some $\varepsilon_{0}$-neighborhood of the point

$\lambda^{(0)}=\left(\lambda_{1}^{(0)}, z\left(\lambda_{1}^{(0)}\right)\right) \in \Lambda \quad \frac{\partial \Psi_{n}}{\partial \lambda_{2}}\left(\lambda_{1}, z\left(\lambda_{2}\right)\right) \neq 0$, then in

an arbitrarily small $\varepsilon_{*}$-neighborhood of that point there exists a continuous differentiable function

$\lambda_{2, *}=\varphi_{*}\left(\lambda_{1}\right)$, which is solution of $(41)$, that is in some bicylindrical domain

$$
\Lambda_{0}=\left\{\left(\lambda_{1}, \lambda_{2}\right) \in \Lambda_{0}:\left|\lambda_{1}-\lambda_{1}^{(0)}\right|<\varepsilon_{1},\left|\lambda_{2}-\lambda_{2}^{(0)}\right|<\varepsilon_{2}\right\}
$$

there exists a connected component of spectrum of the operator-function $\mathrm{A}_{N_{*}}\left(\lambda_{1}, \lambda_{2}\right)\left(\varepsilon_{1}, \varepsilon_{2}\right.$ are small real constants).

Proof. The proof of Theorem is based on Theorems 1, $2[14$, p. 68, 69] and on existence of implicit functions (see, example, [18]). At first we show that the conditions of Theorem 1 [14, p. 68] concerning the existence of discrete spectrum of operator-function $A_{\beta}\left(\lambda_{1}\right)$ follow from the conditions of formulated Theorem. Under the conditions of Theorem the operator $\mathrm{A}\left(\lambda_{1}, \lambda_{2}\right)$ is Fredholm operator with zero index for each $\lambda=\left(\lambda_{1}, \lambda_{2}\right) \in \Lambda$, and the operator-function $\mathrm{A}(\cdot, \cdot): \Lambda \rightarrow \mathrm{L}(E, E)$ is holomorphic. From here follows that at each $\lambda_{1} \in \Lambda_{1, \beta}$ the operator $A_{\beta}\left(\lambda_{1}\right)$, as reduction of the operator $\mathrm{A}\left(\lambda_{1}, \lambda_{2}\right)$, is also Fredholm operator with zero index, and the op- 
erator-function $\mathrm{A}_{\beta}\left(\lambda_{1}\right): \Lambda_{1, \beta} \rightarrow \mathrm{L}(E, E)$ is holomorphic. So, for the operator-function $A_{\beta}\left(\lambda_{1}\right)$ the conditions of Theorem 1 [14, p. 68] are satisfied, from which follows: each point $\lambda_{1}^{(0)} \in S\left(\mathrm{~A}_{\beta}\right)$ is isolated, it is the eigenvalue of the operator $A_{\beta}\left(\lambda_{1}\right)$, the finite-dimensional eigensubspace and finite root subspace correspond to it. Thus, each point $\lambda^{(0)}=\left(\lambda_{1}^{(0)}, z\left(\lambda_{1}^{(0)}\right)\right) \in \Lambda$ is the spectrum point of the operator-function $\mathrm{A}\left(\lambda_{1}, \lambda_{2}\right)$.

In addition, the conditions of Theorem 2 [14, p. 69] are satisfied for the operator-function

$\mathrm{A}_{\beta}\left(\lambda_{1}\right): \Lambda_{1, \beta} \rightarrow \mathrm{L}(E, E)$. From this theorem follows: at $n$ larger than some $n_{0} \in \mathbb{N}$ for each $\lambda_{1}^{(0)} \in S\left(\mathrm{~A}_{\beta}\right)$ there exists a sequence $\left\{\lambda_{1, n}\right\}$ from $\lambda_{1, n} \in S\left(\mathrm{~A}_{\beta, n}\right)$ such that $\lambda_{1, n} \rightarrow \lambda_{1}^{(0)}$. Thus, each point $\left(\lambda_{1}^{(0)}, \lambda_{2}^{(0)}\right)=$ $\left(\lambda_{1}^{(0)}, z\left(\lambda_{1}^{(0)}\right)\right)$ is the eigenvalue of operator-function

$\mathrm{A}_{\beta}\left(\lambda_{1}\right): \Lambda_{1, \beta} \rightarrow \mathrm{L}(E, E)$ and, respectively, the eigenvalue of operator-function $\mathrm{A}(\cdot, \cdot): \Lambda \rightarrow \mathrm{L}(E, E)$. Since $\lambda_{1, n}^{(0)} \in S\left(\mathrm{~A}_{\beta, n}\right)$ is the root of (41), then from here follows that

$$
\Psi_{n}\left(\lambda_{1, n}^{(0)}, z\left(\lambda_{1, n}^{(0)}\right)\right)=0, \quad n \in \mathbb{N} .
$$

From the convergence of sequence $\lambda_{1, n}^{(0)} \in s\left(\mathrm{~A}_{\beta, n}\right)$ to $\lambda_{1}^{(0)} \in s\left(\mathrm{~A}_{\beta}\right)$ follows that for an arbitrarily small number $\varepsilon_{*}>0$ there exists such number $N_{*}>n_{0}$ that $\left|\lambda_{1, N_{*}}^{(0)}-\lambda_{1}^{(0)}\right|<\varepsilon_{*}$ and $\Psi_{N_{*}}\left(\lambda_{1, N_{*}}^{(0)}, z\left(\lambda_{1, N_{*}}^{(0)}\right)\right)=0$.

Let $\lambda_{1}, \lambda_{2}$ be independent variables in the domain $\Lambda$, and $\left(\lambda_{1}^{(0)}, \lambda_{2}^{(0)}\right)=\left(\lambda_{1}^{(0)}, z\left(\lambda_{1}^{(0)}\right)\right) \in \Lambda$ be a spectrum point of the operator-function $A\left(\lambda_{1}, \lambda_{2}\right)$ belonging to $s\left(\mathrm{~A}_{\beta}\right) \subset s(\mathrm{~A})$. Under the conditions of Theorem the functions $\Psi_{n}\left(\lambda_{1}, \lambda_{2}\right)$ are differentiable in the neighborhood of the point $\left(\lambda_{1}^{(0)}, \lambda_{2}^{(0)}\right)=\left(\lambda_{1}^{(0)}, z\left(\lambda_{1}^{(0)}\right)\right)$ and $\frac{\partial \Psi_{N_{*}}}{\partial \lambda_{2}}\left(\lambda_{1, N_{*}}^{(0)}, z\left(\lambda_{1, N_{*}}^{(0)}\right)\right) \neq 0$. In addition the point $\left(\lambda_{1, N_{*}}^{(0)}, z\left(\lambda_{1, N_{*}}^{(0)}\right)\right)$ belongs to $\varepsilon_{*}$-vicinity of the point $\left(\lambda_{1}^{(0)}, \lambda_{2}^{(0)}\right)$. According to the Theorem about implicit function in some neighborhood of point $\left(\lambda_{1}^{(0)}, \lambda_{2}^{(0)}\right)$ there exists the continuous differentiable function $\lambda_{2}=\varphi_{N_{*}}\left(\lambda_{1}\right)$, solving the equation $\Psi_{N_{*}}\left(\lambda_{1}, \lambda_{2}\right)=0$, and $\varphi_{n}\left(\lambda_{1, N_{*}}^{(0)}\right)=z\left(\lambda_{1, N_{*}}^{(0)}\right)$. From here follows existence

of connected spectrum component of the operator-function $\mathrm{A}(\cdot, \cdot): \Lambda \rightarrow \mathrm{L}(E, E)$ in some bicylindrical domain

$$
\Lambda_{0}=\left\{\left(\lambda_{1}, \lambda_{2}\right) \in \Lambda_{0}:\left|\lambda_{1}-\lambda_{1}^{(0)}\right|<\varepsilon_{1},\left|\lambda_{2}-\lambda_{2}^{(0)}\right|<\varepsilon_{2}\right\},
$$

where $\varepsilon_{1}, \varepsilon_{2}$ are small real constants.

Theorem is proved.

Comment. Note that in the case of real parameters $\lambda_{1}$ and $\lambda_{2}$ the presence of a singular point in the equation
$\Psi\left(\lambda_{1}, \lambda_{2}\right)=0$ [19] is one of the sufficient criterions of satisfaction of condition $s(\mathrm{~A}) \neq \Lambda$. The point $\left(\lambda_{1}^{(0)}, \lambda_{2}^{(0)}\right) \in \Lambda$ is a singular point of the curve which is presented by the equation $\Psi\left(\lambda_{1}, \lambda_{2}\right)=0$ when $\frac{\partial \Psi\left(\lambda_{1}^{(0)}, \lambda_{2}^{(0)}\right)}{\partial \lambda_{1}}=0, \frac{\partial \Psi\left(\lambda_{1}^{(0)}, \lambda_{2}^{(0)}\right)}{\partial \lambda_{2}}=0$, and the second order partial derivatives are nonzero:

$$
\begin{aligned}
& \frac{\partial^{2} \Psi\left(\lambda_{1}^{(0)}, \lambda_{2}^{(0)}\right)}{\partial \lambda_{1}^{2}} \neq 0, \frac{\partial^{2} \Psi\left(\lambda_{1}^{(0)}, \lambda_{2}^{(0)}\right)}{\partial \lambda_{2}^{2}} \neq 0, \\
& \frac{\partial^{2} \Psi\left(\lambda_{1}^{(0)}, \lambda_{2}^{(0)}\right)}{\partial \lambda_{1} \partial \lambda_{2}} \neq 0
\end{aligned} .
$$

These derivatives and the third-order derivatives are continuous in the neighborhood of the point $\left(\lambda_{1}^{(0)}, \lambda_{2}^{(0)}\right) \in \Lambda$. If in addition

$$
\left(\frac{\partial^{2} \Psi\left(\lambda_{1}^{(0)}, \lambda_{2}^{(0)}\right)}{\partial \lambda_{1} \partial \lambda_{2}}\right)^{2}-\frac{\partial^{2} \Psi\left(\lambda_{1}^{(0)}, \lambda_{2}^{(0)}\right)}{\partial \lambda_{1}^{2}} \cdot \frac{\partial^{2} \Psi\left(\lambda_{1}^{(0)}, \lambda_{2}^{(0)}\right)}{\partial \lambda_{2}^{2}}<0,
$$

then the point $\left(\lambda_{1}^{(0)}, \lambda_{2}^{(0)}\right)$ is the second order root of equation $\Psi\left(\lambda_{1}, \lambda_{2}\right)=0$. Inside of a sufficiently small radius circle with center at point $\left(\lambda_{1}^{(0)}, \lambda_{2}^{(0)}\right)$ the left part of equation $\Psi\left(\lambda_{1}, \lambda_{2}\right)=0$ becomes zero only at point $\left(\lambda_{1}^{(0)}, \lambda_{2}^{(0)}\right)$, i.e. $\left(\lambda_{1}^{(0)}, \lambda_{2}^{(0)}\right)$ is the isolated point of spectrum.

\subsection{Finding the Connected Components of a Spectrum}

Thus, assuming the existence of discrete spectrum $s\left(\mathrm{~A}_{\beta}\right) \neq \Lambda_{1, \beta}$ and solving an auxiliary one-dimensional spectral problem (42), we find a set of the eigenvalues $\lambda^{(0)}=\left(\lambda_{1}^{(0)}, z\left(\lambda_{1}^{(0)}\right)\right) \in \Lambda$, which belongs also to the spectrum of operator-function $\mathrm{A}(\cdot, \cdot): \Lambda \rightarrow \mathrm{L}(E, E)$. To find the connected components of a spectrum in some neighborhoods of the points $\lambda^{(0)}=\left(\lambda_{1}^{(0)}, z\left(\lambda_{1}^{(0)}\right)\right) \in \Lambda$ we consider the problem on finding the solutions of equation $\Psi_{n}\left(\lambda_{1}, \lambda_{2}\right)=0$, as the problem on finding the implicitly given function $\lambda_{2}=\lambda_{2}\left(\lambda_{1}\right)$ at satisfaction of condition $\partial \Psi_{n}\left(\lambda_{1}^{(0)}, \lambda_{2}^{(0)}\right) / \partial \lambda_{2} \neq 0$ (or $\lambda_{1}=\lambda_{1}\left(\lambda_{2}\right)$ at satisfaction of condition $\left.\partial \Psi_{n}\left(\lambda_{1}^{(0)}, \lambda_{2}^{(0)}\right) / \partial \lambda_{1} \neq 0\right)$, solving the corresponding Cauchy problem

$$
\frac{\mathrm{d} \lambda_{2}}{\mathrm{~d} \lambda_{1}}=-\frac{\partial \Psi_{n}\left(\lambda_{1}^{(0)}, \lambda_{2}^{(0)}\right) / \partial \lambda_{1}}{\partial \Psi_{n}\left(\lambda_{1}^{(0)}, \lambda_{2}^{(0)}\right) / \partial \lambda_{2}},
$$




$$
\lambda_{2}\left(\lambda_{1}^{(0)}\right)=z\left(\lambda_{1}^{(0)}\right) .
$$

Corresponding (38) auxiliary one-dimensional spectral problem has the form

$\mathrm{A}_{M_{n}}\left(\lambda_{1}, z\left(\lambda_{1}\right)\right) x^{(n)} \equiv\left(T_{M_{n}}\left(\lambda_{1}, z\left(\lambda_{1}\right)\right)-I_{M_{n}}\right) x^{(n)}=0$.

Obviously, the eigenvalues of this problem are the roots of equation

$$
\Psi_{M_{n}}\left(\lambda_{1}\right)=\operatorname{det}\left(T_{M_{n}}\left(\lambda_{1}, z\left(\lambda_{1}\right)\right)-I_{M_{n}}\right)=0 .
$$

Solving the problem (45), (46) in some neighborhood of the point $\lambda^{(i)}=\left(\lambda_{1}^{(i)}, z\left(\lambda_{2}^{(i)}\right)\right) \in \Lambda$, we find the $i$-th connected component of spectrum of the operator-function $\mathrm{A}_{M_{n}}\left(\lambda_{1}, \lambda_{2}\right)$.

Return to finding the solutions of (33), in which $c_{1}$, $c_{2}$ are real spectral parameters. Let $\left(c_{1}, c_{2}\right) \in \Lambda_{c}$, $\Lambda_{c}=\Lambda_{c_{1}} \times \Lambda_{c_{2}}$, where $\Lambda_{c_{i}}=\left\{c_{i} \in \Lambda_{c_{i}}: 0<c_{i}<r_{c}\right\}$. By direct check we set that for arbitrary values of parameters $\left(c_{1}, c_{2}\right) \in \Lambda_{c}$ the function

$$
\varphi_{0}(Q, \mathbf{c})=f_{0}(Q, \mathbf{c}),
$$

is one of the eigenfunctions, where $f_{0}(Q, \mathbf{c})$ is the initial solution of (7). Write the necessary in what follows equation

$$
\begin{aligned}
& \psi(Q)=T^{*}(\mathbf{c}) \psi \\
& \equiv\left(F(Q)-f_{0}(Q, \mathbf{c})\right) \cdot \iint_{\bar{\Omega}} K\left(Q, Q^{\prime}, \mathbf{c}\right) \frac{\psi\left(Q^{\prime}\right)}{f_{0}\left(Q^{\prime}, \mathbf{c}\right)} \mathrm{d} Q^{\prime}
\end{aligned}
$$

conjugate with (33). For arbitrary $\left(c_{1}, c_{2}\right) \in \Lambda_{c}$ the function

$$
\psi_{0}(Q, \mathbf{c})=F(Q)-f_{0}(Q, \mathbf{c})
$$

is one of the eigenfunctions of (33).

The existence of distinct from identical to zero solutions of (33) for arbitrary $\left(c_{1}, c_{2}\right) \in \Lambda_{c}$ indicates that there is a connected component of spectrum, coinciding with the domain $\Lambda_{c}$. So, the condition of Theorem 2: $s(\tilde{\mathrm{A}}) \neq \Lambda_{c}$ is not satisfied. To satisfy this condition we exclude eigenfunctions (49), (51) from the kernel of integral equation (33). Consider the equation

$$
\varphi(Q, \mathbf{c})=\tilde{T}(\mathbf{c}) \varphi \equiv \iint_{\bar{\Omega}} \tilde{\mathrm{K}}\left(Q, Q^{\prime}, \mathbf{c}\right) \varphi\left(Q^{\prime}\right) \mathrm{d} Q^{\prime},
$$

where

$$
\begin{aligned}
\tilde{\mathrm{K}}\left(Q, Q^{\prime}, \mathbf{c}\right)= & \frac{F\left(Q^{\prime}\right)-f_{0}\left(Q^{\prime}, \mathbf{c}\right)}{f_{0}\left(Q^{\prime}, \mathbf{c}\right)} K\left(Q, Q^{\prime}, \mathbf{c}\right) \\
& -\frac{\psi_{0}(Q, \mathbf{c}) \varphi_{0}\left(Q^{\prime}, \mathbf{c}\right)}{\left\|\psi_{0}\right\| \cdot\left\|\varphi_{0}\right\|}
\end{aligned}
$$

From the Schmidt Lemma [16, p. 132] follows that $\mu=1$ is not characteristic value of (52) for any value $\left(c_{1}, c_{2}\right)$, that is $\varphi_{0}(Q, \mathbf{c})$ is not an eigenfunction of this equation. Thereby the connected component coinciding with the domain $\Lambda_{c}$ and corresponding to the function $\varphi_{0}(Q, \mathbf{c})$ is excluded from the spectrum of operator.
Using (8) we are sure that for the kernel of operator $\tilde{T}(\mathbf{c})$ is fulfilled the inequality

$$
\begin{aligned}
& \iint_{\bar{\Omega}} \iint_{\bar{\Omega}}\left|\tilde{\mathrm{K}}\left(Q, Q^{\prime}, \mathbf{c}\right)\right|^{2} \mathrm{~d} Q \mathrm{~d} Q^{\prime} \\
& \leq \frac{1}{\alpha^{2}}\left(\frac{c_{1} c_{2}}{(2 \pi)^{2}}\right)^{2} \mu^{3}(\bar{\Omega}) \iint_{\bar{\Omega}} \frac{\psi_{0}^{2}(Q, \mathbf{c})}{\varphi_{0}^{2}(Q, \mathbf{c})} \mathrm{d} Q \\
& +\frac{c_{1} c_{2}}{(2 \pi)^{2}}\left(\iint_{\bar{\Omega}} \psi_{0}(Q, \mathbf{c}) \mathrm{d} Q\right)^{2}+\mu^{2}(\bar{\Omega})<+\infty .
\end{aligned}
$$

Here $\mu(\bar{\Omega})$ is the measure of the domain $\bar{\Omega}$. From the obtained inequality follows that $\tilde{T}(\mathbf{c})$ is the Fredholm operator with zero index [201. Moreover, it is a completely continuous operator in space $L_{2}(\Omega)$ [21].

Functions entering in the kernel of the operator (35), admit the analytic continuation into the complex domain $\Lambda_{c}$, if $c_{1}$ and $c_{2}$ are assumed as complex parameters. Holomorphy of operator-function $\mathrm{A}\left(c_{1}, c_{2}\right)=$ $=\tilde{T}\left(c_{1}, c_{2}\right)-I$ follows [14] from existence of partial derivatives $\frac{\partial \mathrm{A}\left(c_{1}, c_{2}\right)}{\partial c_{i}} \quad(i=1,2)$ at arbitrary point $\left(c_{1}^{(0)}, c_{2}^{(0)}\right) \in \Lambda_{c}$ owing to continuity of the kernel $\tilde{\mathrm{K}}\left(Q, Q^{\prime}, \mathbf{c}\right)$ according to a set of their variables in the domain $\bar{\Omega} \times \bar{\Omega} \times \bar{\Lambda}_{c}$ and existence and continuity of partial derivatives $\frac{\partial \mathrm{K}\left(c_{1}, c_{2}\right)}{\partial c_{i}} \quad(i=1,2)$, what is easy to verify.

Putting in (52), $c_{2}=\beta c_{1}$, we shall consider the onedimensional spectral problem

$$
\varphi(Q)=\tilde{\tilde{T}}\left(c_{1}\right) u \equiv \iint_{G} \tilde{\tilde{\mathrm{K}}}\left(Q, Q^{\prime}, c_{1}\right) \varphi\left(Q^{\prime}\right) \mathrm{d} Q^{\prime},
$$

where $\tilde{\tilde{\mathrm{K}}}\left(Q, Q^{\prime}, c_{1}\right)=\tilde{\mathrm{K}}\left(Q, Q^{\prime}, c_{1}, \beta c_{1}\right)$. Since $\tilde{\tilde{T}}\left(c_{1}\right)$ is reduction of the operator $\tilde{T}(c)$, from here follows that $\tilde{\tilde{T}}\left(c_{1}\right)$ is the Fredholm operator with zero index for any $c_{1} \in \Lambda_{1, \beta}$, and the operator-function $\tilde{\mathrm{A}}(\cdot) \equiv(\tilde{\tilde{T}}(\cdot)-I): \Lambda_{1} \rightarrow \mathrm{L}(E, E)$ is holomorphic.

If $s(\tilde{\mathrm{A}}) \neq \Lambda_{1}$, from holomorphy of operator-function and from the Fredholm property of the kernel $\widetilde{\mathrm{K}}\left(Q, Q^{\prime}, c_{1}\right)=\mathrm{K}\left(Q, Q^{\prime}, c_{1}, \beta c_{1}\right)$ satisfaction of the conditions of Theorem 2 follows. In accordance with this Theorem every point $c_{1}^{(0)} \in S(\tilde{\mathrm{A}})$ is isolated and it is the eigenvalue of (54). Respectively, the points $\left(c_{1}^{(0)}, c_{2}^{(0)}\right)=$ $=\left(c_{1}^{(0)}, \beta c_{1}^{(0)}\right)$ are eigenvalues of (54). To find the spectrum connected components (52) in the vicinities of points $\left(c_{1}^{(0)}, c_{2}^{(0)}\right)$ we solve the Cauchy problem (45) 
and (46), using the found solutions of auxiliary problem (54) as initial conditions.

\subsection{Numerical Finding the Eigenvalues of the Problem}

We shall construct algorithms to find numerically the eigenvalues of (54) what corresponds to auxiliary problem (43). Consider some convergent cubature process [14]

$$
\iint_{\bar{\Omega}} x(Q) \mathrm{d} Q=\sum_{j=1}^{n} a_{j n} x\left(Q_{j n}\right)+\phi_{n}(x),(n \in \mathbb{N})
$$

with coefficients $a_{j n} \in \mathbb{R}$ and nodes $Q_{j n} \in \bar{\Omega}$ $(j=1 \div n)$. We reject the remainder term $\phi_{n}(x)$ in (55) and replace integral in (52) by it. Giving the variable $Q$ values $Q=Q_{i n} \quad(i=1 \div n)$, we have the homogeneous system of linear algebraic equations concerning

$u_{1 n}, \cdots, u_{n n}$ :

$$
u_{i n}=\tilde{\tilde{T}}_{M_{n}}\left(c_{1}\right) u_{n} \equiv \sum_{j=1}^{n} a_{j n} \tilde{\mathrm{K}}\left(Q_{i n}, Q_{j n}, c_{1}\right) u_{j n},(i=1 \div n) \text { (56) }
$$

where $u_{i n}=u\left(Q_{i n}\right)$. Solving the eigenvalue problem (56) in the finite-dimensional spaces $E_{n}=C\left(\bar{\Omega}_{n}\right)$, we find approximate eigenvalues, convergent to exact solutions of the problem (54) as $n \rightarrow \infty$.

Finding the eigenvalues of (56) is reduced, in particular, to finding the roots of equation

$$
\Psi_{n}\left(c_{1}\right)=\operatorname{det}\left(\tilde{\tilde{T}}_{M_{n}}\left(c_{1}\right)-I_{n}\right)=0 .
$$

These solutions of equation we denote as $c_{1}^{(i)}$.

Return to two-dimensional spectral problem (52). Applying the (55) to (52), we obtain a system of linear equations similar to (56)

$$
\begin{array}{r}
u_{i n}=T_{M_{n}}\left(c_{1}, c_{2}\right) u \equiv \sum_{j=1}^{n} a_{j n} \tilde{\mathrm{K}}\left(Q_{i n}, Q_{j n}, c_{1}, c_{2}\right) u_{j n} \\
(i=1 \div n) .
\end{array}
$$

Finding the solutions of equations

$$
\Psi_{n}\left(c_{1}, c_{2}\right)=\operatorname{det}\left(T_{M_{n}}\left(c_{1}, c_{2}\right)-I_{n}\right)=0
$$

we consider as a problem on finding the implicitly given function $c_{2}=c_{2}\left(c_{1}\right)$, reducing it to the Cauchy problem (45), (46). Since to each isolated root of this equation corresponds eigenvalue $\left(c_{1}^{(i)}, c_{2}^{(i)}\right)=\left(c_{1}^{(i)}, \beta c_{1}^{(i)}\right)$ of problem (58) we use solutions of (57) as the initial conditions (46). Thus, we determine the initial conditions (46) for the Cauchy problem as $c_{2}^{(i)}\left(c_{1}^{(i)}\right)=\beta c_{1}^{(i)}$. If $\partial \Psi_{n}\left(c_{1}, c_{2}\right) / \partial c_{2} \neq 0$ then solving the problem (45) (46) in each vicinity of points $c_{1}^{(i)}$, we find the differentiable function $c_{2}=\gamma_{i}\left(c_{1}\right)$ which satisfies the condition $\gamma\left(c_{1}^{(i)}\right)=\beta c_{1}^{(i)}$.
In the case, when $\left(c_{1}^{(i)}, \beta c_{1}^{(i)}\right)$ is a real eigenvalue of the problem (57), $c_{2}=\gamma_{i}\left(c_{1}\right)$ are real differentiable functions describing in the vicinity of points $\left(c_{1}^{(i)}, \beta c_{1}^{(i)}\right)$ some smooth curves. That is, in this case the equations (52) and (33) have a linear spectrum, respectively.

Thus, solving the problems (57) and (58), we find a set of values of parameters $\left(c_{1}, c_{2}\right) \in \Lambda_{c}$ at which the branching of complex solutions of Equation (7) from the real initial solution $f_{0}(Q, \mathbf{c})$ at $\mu>0, v>0$ is possible. Functional $\left\{\sigma_{\alpha}(U)\right\}$ has smaller values on branchingoff solutions than on the solution $f_{0}(Q, \mathbf{c})$.

\section{Algorithm of Finding the Solutions of Nonlinear Equation. Numerical Examples}

Present one of iterative processes for numerical finding the solutions of system (13), based on the successive approximations method:

$$
\left\{\begin{array}{l}
u_{n+1}=\alpha^{-1}\left(I+\alpha^{-1} B_{11}\right)^{-1} B_{12}\left(u_{n}, v_{n}\right), \\
v_{n+1}=\alpha^{-1}\left(I+\alpha^{-1} B_{21}\right)^{-1} B_{22}\left(u_{n}, v_{n}\right),(n=0,1, \cdots) .
\end{array}\right.
$$

Before it was shown that the inverse operators $\left(I+\alpha^{-1} B_{11}\right)^{-1}$ and $\left(I+\alpha^{-1} B_{21}\right)^{-1}$ exist and are limited.

In the case of even on both arguments function $F\left(s_{1}, s_{2}\right)$ and symmetric domains $G$ and $\Omega$ at execution of iterative process (60) it is appropriate to use the invariance property of the integral operators $B_{1}(u, v)$ and $B_{2}(u, v)$ in the system (13) concerning the type of parity of functions $u\left(s_{1}, s_{2}\right), v\left(s_{1}, s_{2}\right)$. Functions $u$ and $v$ having some type of parity on the corresponding argument belong to the invariant sets $U_{i j}$ and $V_{k l}$ of the space $C(\bar{\Omega})$ where the indices $i, j, k, l$ take values 0 or 1 . In particular, if $u\left(s_{1}, s_{2}\right) \in U_{01}$ then

$u\left(-s_{1}, s_{2}\right)=u\left(s_{1}, s_{2}\right), u\left(s_{1},-s_{2}\right)=-u\left(s_{1}, s_{2}\right)$. By the direct check we are convinced that there are such inclusions:

$$
\begin{aligned}
& B_{1}\left(U_{i j} \cup V_{k l}\right) \subset U_{i j}, B_{2}\left(U_{i j} \cup V_{k l}\right) \subset V_{k l}, \\
& \mathbf{B}\left(U_{i j} \cup V_{k l}\right) \subset U_{i j} \cup V_{k l}
\end{aligned}
$$

From these relations, in particular, follows the possibility of existence of fixed points of operator $\mathbf{B}$ belonging to the corresponding invariant set, that is to solutions of (13) and, respectively, (7).

Substitute into (10) the function $\arg f_{n}(Q)=$ $=\operatorname{arctg}\left(v_{n}(Q) / u_{n}(Q)\right)$ obtained on the basis of successive approximations (60). As a result we have a sequence of function values which we denote as $\left\{U_{n}\right\}$. For this sequence the Theorem 4.3.2 and corollary 4.3.1 [4], and 
the Theorem 4 from [22] are valid. From here follows that the sequence $\left\{U_{n}\right\}$ is relaxation for functional (7) and the numerical sequence $\left\{\sigma_{\alpha}\left(U_{n}\right)\right\}$ is convergent.

Consider the numerical examples of approximation of even on both arguments function

$F\left(s_{1}, s_{2}\right)=\left|\sin \left(\pi \mathrm{s}_{1}\right)\right|\left|\sin \left(\pi \mathrm{s}_{2}\right)\right|$ (Figure 1) in the domain $\bar{\Omega}=\left\{\left(s_{1}, s_{2}\right):\left|s_{1}\right| \leq 1,\left|s_{2}\right| \leq 1\right\}$.

In Figure 2 in logarithmic scale the values of functional $\sigma_{\alpha}(U)$, which it takes on different types of solutions of system of (13) at change of the parameters $c_{1}, c_{2}$ on the beams $c_{2}=0.8 c_{1}$ are presented. The curve 1 corresponds to the initial solution $f_{0}\left(s_{1}, s_{2}\right)$.

The curve 2 is a branchino-nff solution at point $\left(c_{1}^{(1)}, c_{2}^{(1)}\right)=(2.345,1.876)$ with the property $\arg f\left(s_{1},-s_{2}\right)=-\arg f\left(s_{1}, s_{2}\right)$. From the analysis of

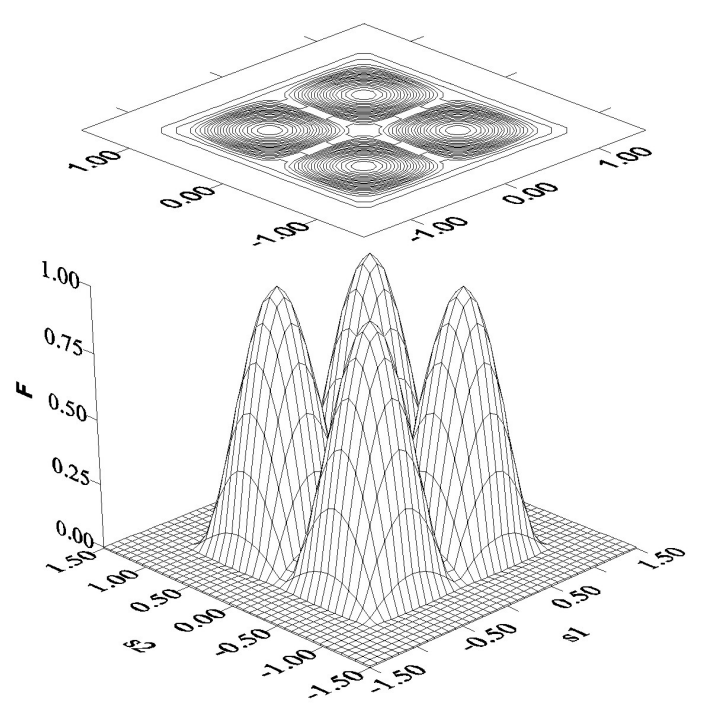

Figure 1. The function $F\left(s_{1}, s_{2}\right)=\left|\sin \left(\pi s_{1}\right)\right|\left|\sin \left(\pi s_{2}\right)\right|$ given in the domain $\bar{\Omega}=\left\{\left(s_{1}, s_{2}\right):\left|s_{1}\right| \leq 1,\left|s_{2}\right| \leq 1\right\}$.

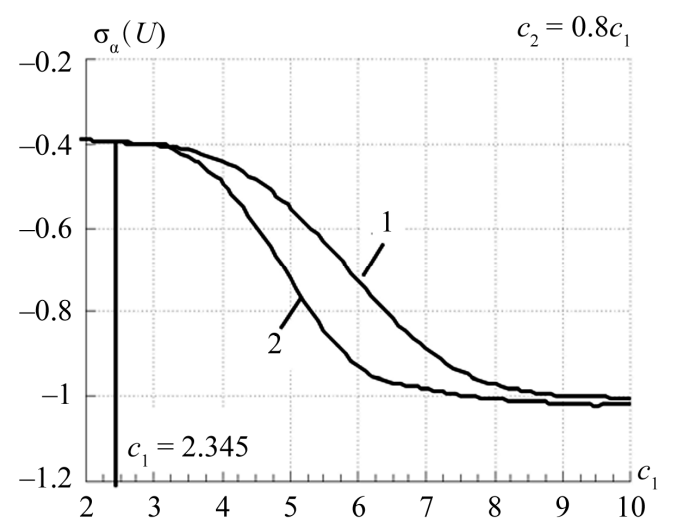

Figure 2. The values of functional on initial and branchingoff solutions.
Figure follows that branching-off solutions at point $\left(c_{1}^{(1)}, c_{2}^{(1)}\right)$ are more effective compared with initial solution $f_{0}$, since the functional $\sigma_{\alpha}(U)$ accepts smaller values on branching-off solutions than on initial.

The points of possible branching of solutions of (7) (spectral lines of (33)) for given

$F\left(s_{1}, s_{2}\right)=\left|\sin \left(\pi \mathrm{s}_{1}\right)\right|\left|\sin \left(\pi \mathrm{s}_{2}\right)\right|$ are shown in Figure 3.

Figures 4 and $\mathbf{5}$ present $\left|f\left(s_{1}, s_{2}\right)\right|$ and $\arg f\left(s_{1}, s_{2}\right)$ of approximate function corresponding to the branching-off solution of system of Equations (13) at $c_{1}=8$ and $\mathrm{c}_{2}=6.4$.

Correspoding to this solution the functions $|U(x, y)|$ and $\arg U(x, y)$ of the Fourier integral prototype in a spatial image and image by the level lines are shown, respectively, in Figures 6 and 7.

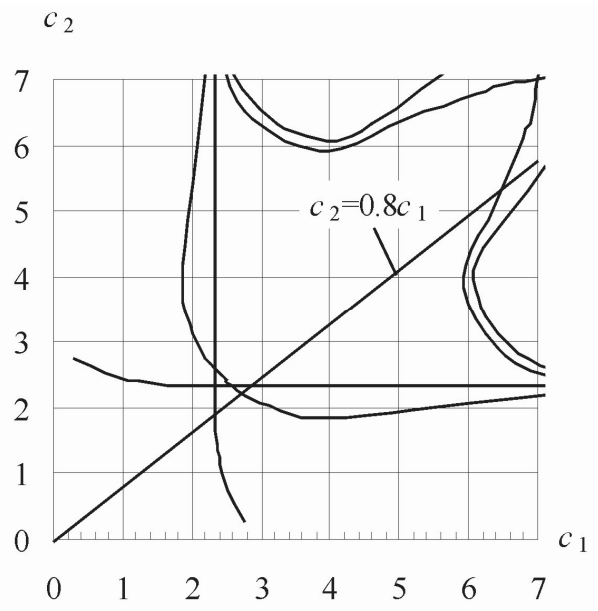

Figure 3. Points of possible branching of solutions (spectral lines) for given $F\left(s_{1}, s_{2}\right)=\left|\sin \left(\pi s_{1}\right)\right|\left|\sin \left(\pi s_{2}\right)\right|$.
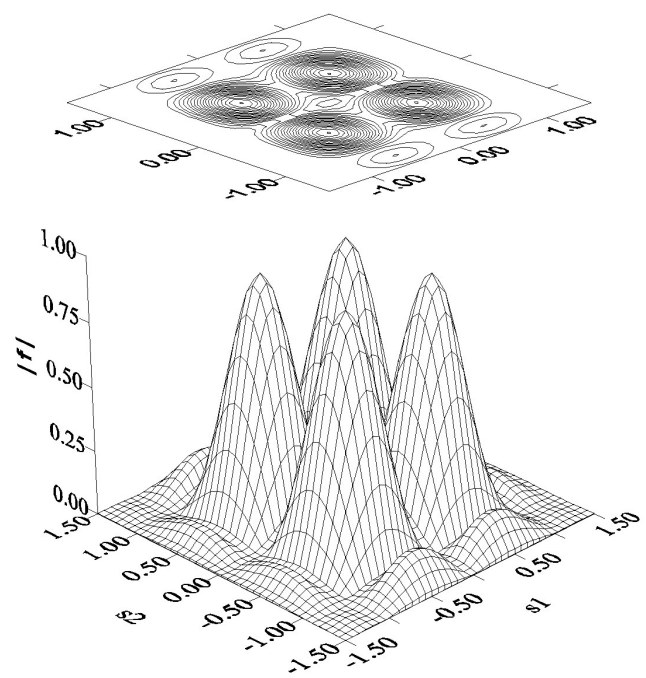

Figure 4. The modulus of approximation function. 


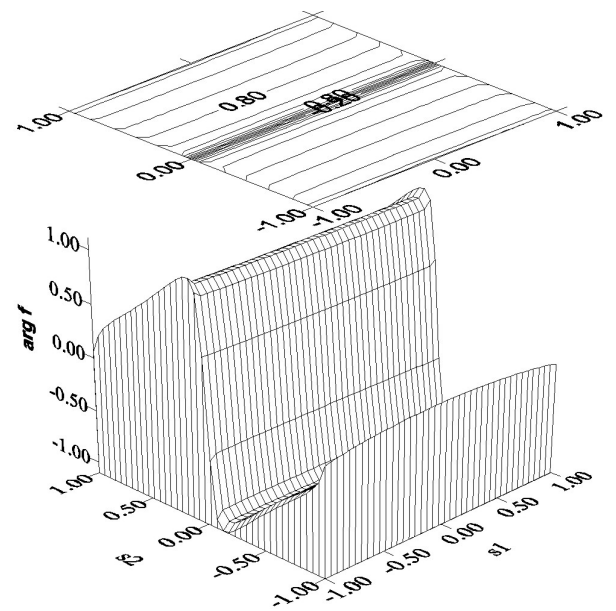

Figure 5. The argument of approximation function.
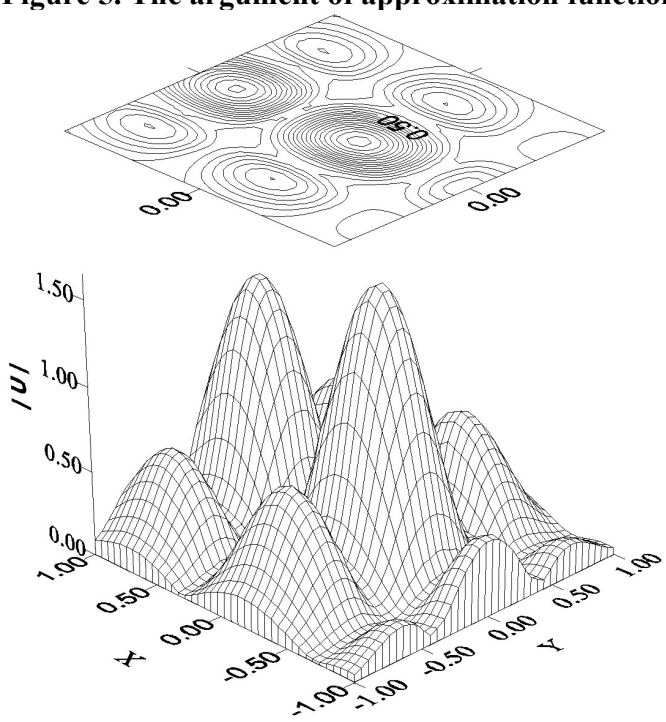

Figure 6. The amplitude of Fouier integral prototype.
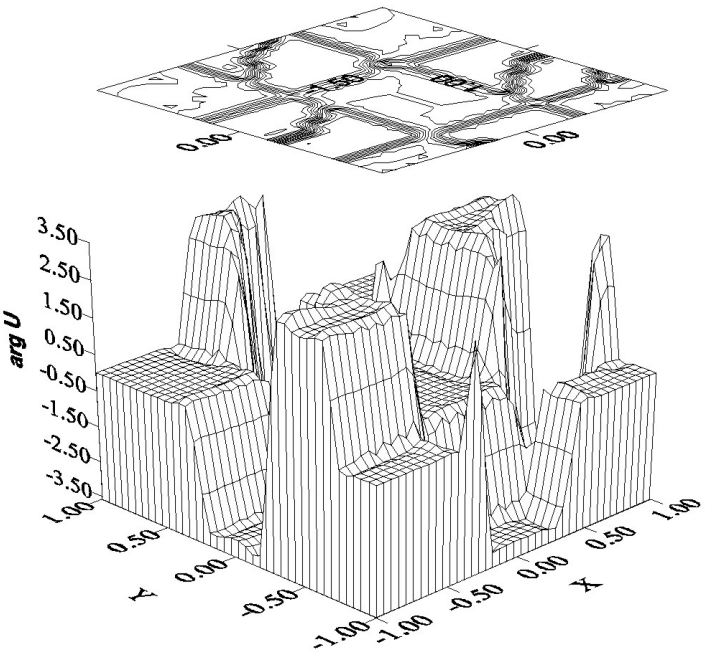

Figure 7. The argument of Fouier integral prototype.
As we see in these figures, the function $|U(x, y)|$ is a nonsymmetric relatively to the center of the domain $G$ along the axis $O X$, and $\arg U(x, y)$ accepts the value 0 or $\pi$ in the corresponding subdomains of the domain $G$, that is function $U(x, y)$ is real. Thus, from the analysis of Figures follows that the given symmetric function $F\left(s_{1}, s_{2}\right)$ (even on both arguments) on the beam $c_{2}=0.8 c_{1}$ at $c_{1}>c_{1}^{(1)}$ is approximated effectively by the optimal choice of nonsymmetrical by modulus Fourier integral prototypes, that is the functions $|U(x, y)|$.

\section{To Selection of Parameter $\alpha$}

We shall present some argumentations concerning the choice of the weight parameter of regularization $\alpha$ in the optimization criteria (4). Many of works (see, [23-26]) are devoted to this question. Efficient algorithms of solving this problem are developed mainly for linear operator equations of the type

$$
L u=F_{\delta}
$$

with approximate right part $F_{\delta}$, in which a priori the error $\delta$ is known. Their detailed definition is given in $[23,25]$. The principle of residual is the most applicable one in practice. Here we choose such number $\alpha_{*}$ for which with the necessary accuracy the equality

$$
\left\|L u-F_{\delta}\right\|=\delta
$$

is executed ( $u$ is a minimum point of smoothing functional defined on $H_{U}$ ) dependent on the parameter $\alpha$. As shown in [1], the principle of residual (63) to determine the parameter $\alpha$ in the case of nonlinear operator $L$ can be applied when $L$ is a convex operator. Generally, the residual (63) may be discontinuous or nonmonotonous function with respect to parameter $\alpha$. Therefore Equation (62) can not have any solution or have a set of solutions.

The error $\delta$ is unknown a priori, as a rule, in the problems of nonlinear approximation. Decrease of parameter $\alpha$ in the functional $\sigma_{\alpha}(U)$ reduces the requirements to the norm $\|U\|$. As a result, the norm of the Fourier integral prototype minimizing the functional $\sigma_{\alpha}(U)$, inversely depend on $\alpha$. At reduction $\alpha$ the accuracy of approximation in the limits of the domain $\bar{\Omega}$, as rule. increases. but the value of function $\left|f\left(s_{1}, s_{2}\right)\right|$ outside this domain increases also.

At concrete calculating the parameter $\alpha$ can be selected on the basis of some physical argumentations and numerical experiments. In particular, in the antenna synthesis problems the parameter $\alpha$ can be selected from the satisfaction of equal energy condition [4]

$$
\left\|U_{\alpha}\right\|_{L_{2}(G)}^{2}=\|F\|_{L_{2}(\Omega)}^{2} .
$$


A numerical example of dependence of solutions of (7) on the value of parameter $\alpha$ at approximation of the function $F\left(s_{1}, s_{2}\right)=\cos \frac{\pi s_{1}}{2}\left|\sin \pi s_{2}\right|$ is the proof of the above presented arguments. In Figure 8 are given the values of approximate function $\left|f\left(s_{1}, s_{2}\right)\right|$ in the section $s_{1}=0$. From the analysis of the Figure, we see that the quality of approximation of the given function on the interval $-1 \leq s_{2} \leq 1$ increases when parameter $\alpha$ decreases, while $\left|f\left(0, s_{2}\right)\right|$ increases outside this interval.

\section{Conclusions}

Note the main results and problems arising at investigations of the considered class of problems:

1) The method of nonlinear approximation of finite nonnegative functions with respect to two variables by the modulus of double Fourier transform with use the smoothing functionals is developed in the work.

2) It is shown that non-uniqueness and branching of solutions is characteristic for this class of problems. The numerical method of solving the two-parametric nonlinear spectral problem enabling to find the branching lines of solutions of Hammerstein type nonlinear two-dimensional integral Equation (7) is proposed to study the non-uniqueness of solutions dependent on the value of parameters $c_{1}, c_{2}$ entering the Fourier integral.

3) At finding the solutions of system of Equations (13) by the successive approximations method (60) in the case of even by both arguments (one argument) function $F\left(s_{1}, s_{2}\right)$ to obtain the solution of concrete type it is necessary to select the initial approximation belonging to the corresponding invariant set of nonlinear operators

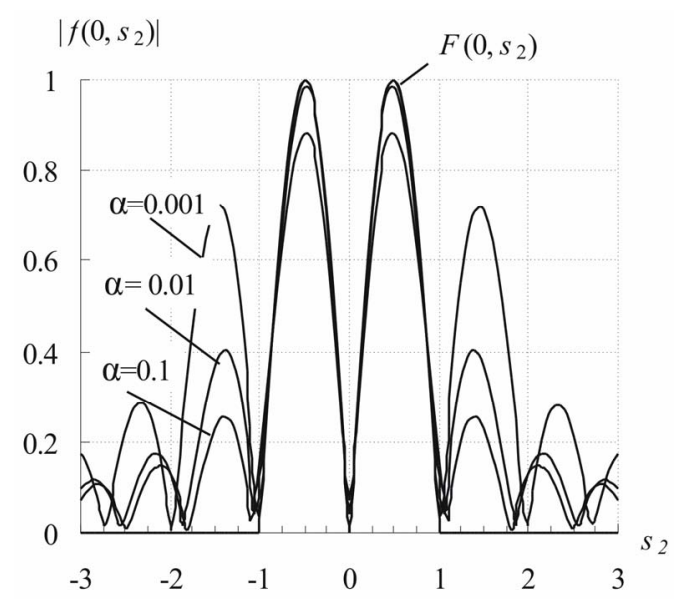

Figure 8. The modulus of approximate function $F\left(s_{1}, s_{2}\right)=\cos \frac{\pi s_{1}}{2}\left|\sin \pi s_{2}\right|$ in the section $s_{2}=0$ corresponding to various regularization parameters $\alpha$.
$B_{1}, B_{2}(61)$.

4) Investigations of branching of existing solutions dependent on physical parameters $c_{1}, c_{2}$ entering the Fourier integral are the main difficulty at solving this class of problems. As follows from the presented researches, in particular, in $[4,13]$, for a special case when $F\left(s_{1}, s_{2}\right)=F_{1}\left(s_{1}\right) \cdot F_{2}\left(s_{2}\right)$, the quantity of existing solutions with increase of parameters $c_{1}, c_{2}$ significantly increases. Note, that in many practical applications, particularly in the synthesis problems of radiating systems, obtaining the best approximation to the given function $F\left(s_{1}, s_{2}\right)$ at concrete values of parameters $c_{1}, c_{2}$ is important. It allows to limit oneself to investigations of few first points (lines) of branching.

5) Obtaining the complete answer about exact quantity of existing solutions of (7) at concrete values of the parameters $c_{1}, c_{2}$ are the subject of separate studies.

\section{References}

[1] A. N. Tikhonov and V. Y. Arsenin, "The Methods of Solution of Incorrect Problems," Nauka, Moscow, 1979.

[2] P. Savenko and M. Tkach, "Numerical Approximation of Real Finite Nonnegative Function by the Modulus of Descrete Fourier Transform," Applied Mathematics, Vol. 1, No. 1, 2010, pp. 65-75. doi:10.4236/am.2010.11008

[3] E. G. Zelkin and S. I. Solov'yev, "Methods of Synthesis of Antennas: Phased Antenna Arrays and Antennas with Continuous Aperture," Sovet radio, Moscow, 1980.

[4] P. O. Savenko, "Nonlinear Problems of Radiating Systems Synthesis (Theory and Methods of the Solution)," Institute for Applied Problems in Mechanics and Mathematics, Lviv, 2002.

[5] A. N. Kolmogorov and S. V. Fomin, "Elements of Functions Theory and Functional Analysis," Nauka, Moscow, 1968.

[6] M. M. Vainberg and V. A. Trenogin, "Theory of Branching of Solutions of Nonlinear Equations," Nauka, Moscow, 1969.

[7] V. A. Trenogin, "The Functional Analysis," Nauka, Moscow, 1980.

[8] M. A. Krasnoselskii, G. M. Vainikko and P. P. Zabreiko, “Approximate Solution of Operational Equations," Nauka, Moscow, 1969.

[9] L. V. Kantorovich and G. P. Akilov, "The Functional Analysis," Nauka, Moscow, 1977.

[10] I. I. Liashko, V. F. Yemel'ianov and A. K. Boyarchuk, "Bases of Classical and Modern Mathematical Analysis," Vysshaya Shkola Publishres, Kyiv, 1988.

[11] S. G. Mikhlin, "The Direct Methods in Mathematical Physics," Gosudarstvennoje Izdatelstvo Tekhnicheskoy Literatury, Moscow-Leningrad, Leningrad, Moscow, 1950.

[12] G. I. Marchuk, "The Methods of Calculus Mathematics," 
Nauka, Moscow, 1977.

[13] P. O. Savenko, "The Branching of Solutions of Antennas Synthesis Problems According to the Given Amplitude Directivity Pattern with Use of Regularization Functionals," Izvestija Vysshykh Uchebnykh Zavedeniy Radioelektronica, Vol. 39, No. 2, 1996, pp. 35-50.

[14] G. M. Vainikko, "Analysis of Discretized Methods," Tartuskiy Gosudarstvennyy Universitet, Tartu, 1976.

[15] P. A. Savenko and L. P. Protsakh, "Implicit Function Method in Solving a Two-dimensional Nonlinear Spectral Problem," Russian Mathematics (Izv. VUZ), Vol. 51, No. 11, 2007, pp. 40-43.

[16] M. M. Vainberg and V. A. Trenogin, "Theory of Branching of Solutions of Nonlinear Equations," Nauka, Moscow, 1969.

[17] I. G. Petrovskii, "Lectures on the Theory of Ordinary Differential Equations," Nauka, Moscow, 1970.

[18] V. I. Smirnov, "Course of High Mathematics, vol. 1," Nauka, Moscow, 1965.

[19] A. Gursa, "Course of Mathematical Analysis, Vol. 1, Part 1," Gosudarstvennoje Tekhniko-teoreticheskoje Izdata- lelstvo, Leningrad, Moscow, 1933.

[20] P. P. Zabreiko, A. I. Koshelev and M. A. Krasnoselskii, "Integral Equations," Nauka, Moscow, 1968.

[21] S. G. Mikhlin, "Variational Methods in Mathematical Physics," Nauka, Moscow, 1970.

[22] P. A. Savenko, "Numerical Solution of a Class of Nonlinear Problems in Synthesis of Radiating Systems," Computational Mathematics and Mathematical Physics, Vol. 40, No. 6, 2000, pp. 889-899.

[23] A. F. Verlan and V. S. Sizikov, "Integral Equations: Methods, Algorithms, Programs. Reference Textbook," Naukova Dumka, Kyiv, 1986.

[24] V. A. Morozov, "Regularizing Methods of Solving the Incorrect Problems," Nauka, Moscow, 1987.

[25] A. N. Tikhonov, A. V. Goncharskyi, V. V. Stepanov and A. G. Yagola, "Regularizing Algoritms and a Priori Information," Nauka, Moscow, 1983.

[26] A. G. Yagola, "About Selection of Regularizing Parameter in the Solution of Incorrect Problems in Reflexive Spaces," Journal of Computational Mathematics and Mathematical Physics, Vol. 20, No. 3, 1980, pp. 586-596. 